\title{
Improved estimates for the Ginzburg-Landau equation: the elliptic case
}

\author{
FAbrice Bethuel, Giandomenico Orlandi And Didier Smets
}

\begin{abstract}
We derive estimates for various quantities which are of interest in the analysis of the Ginzburg-Landau equation, and which we bound in terms of the $G L$-energy $E_{\varepsilon}$ and the parameter $\varepsilon$. These estimates are local in nature, and in particular independent of any boundary condition. Most of them improve and extend earlier results on the subject.
\end{abstract}

Mathematics Subject Classification (2000): 35J60 (primary); 35B40, 35Q40, 31B35, 46E35 (secondary).

\section{Introduction}

Let $\Omega$ be a smooth bounded domain in $\mathbb{R}^{N}, N \geq 2$. For $0<\varepsilon<1$ we consider the complex elliptic Ginzburg-Landau equation

$$
-\Delta u_{\varepsilon}=\frac{1}{\varepsilon^{2}} u_{\varepsilon}\left(1-\left|u_{\varepsilon}\right|^{2}\right) \quad \text { on } \Omega
$$

for maps $u_{\varepsilon}: \Omega \rightarrow \mathbb{C}$. This equation is the Euler-Lagrange equation for the Ginzburg-Landau ( $G L-)$ energy functional

$$
E_{\varepsilon}\left(u_{\varepsilon}\right)=\int_{\Omega} e_{\varepsilon}\left(u_{\varepsilon}\right) \equiv \int_{\Omega} \frac{\left|\nabla u_{\varepsilon}\right|^{2}}{2}+\frac{\left(1-\left|u_{\varepsilon}\right|^{2}\right)^{2}}{4 \varepsilon^{2}},
$$

and we will be concerned only with solutions $u_{\varepsilon}$ with finite $G L$-energy.

The purpose of this paper is to derive estimates for various quantities which are of interest in the analysis of $(\mathrm{GL})_{\varepsilon}$, and which will be bounded in terms of the $G L$ energy $E_{\varepsilon}$ and the parameter $\varepsilon$. These estimates are of local nature, and in particular independent of any boundary condition. Most of them extend and generalize earlier results on the subject.

The $G L$-energy has two components: the kinetic energy $\frac{|\nabla u|^{2}}{2}$ on one side, and the potential energy $V_{\varepsilon}(u) \equiv \frac{\left(1-|u|^{2}\right)^{2}}{4 \varepsilon^{2}}$ on the other. Our first result yields an Pervenuto alla Redazione il 17 settembre 2004 e in forma definitiva l'8 aprile 2005. 
"improved" local bound for the integral of the potential, and shows that, for suitable energy regimes, it is of lower order.

Theorem 1.1. Let $\Omega=B_{1}$ and $u_{\varepsilon}$ be a solution to $(G L)_{\varepsilon}$. We have

$$
\int_{B_{1 / 2}} V_{\varepsilon}\left(u_{\varepsilon}\right) \leq C_{0} \frac{E_{\varepsilon}\left(u_{\varepsilon}\right)}{|\log \varepsilon|} \log \left(2+\frac{E_{\varepsilon}\left(u_{\varepsilon}\right)}{|\log \varepsilon|}\right),
$$

where $C_{0}$ is some constant depending on $N$.

Estimate (1.1) provides some non trivial information only if the energy is not too large, more precisely only if

$$
E_{\varepsilon}\left(u_{\varepsilon}\right) \leq \varepsilon^{-1 / C_{0}}|\log \varepsilon|
$$

On the other hand, it is well-known ${ }^{(1)}$ that there exist real-valued solutions to (GL) $\varepsilon$ such that the "energy balance"

$$
\frac{\left|\nabla u_{\varepsilon}\right|^{2}}{2}=\frac{\left(1-\left|u_{\varepsilon}\right|^{2}\right)^{2}}{4 \varepsilon^{2}}
$$

holds pointwise, and such that $E_{\varepsilon}\left(u_{\varepsilon}\right)$ is of order $\varepsilon^{-1}$. In this case, $\log \left(2+\frac{E_{\varepsilon}\left(u_{\varepsilon}\right)}{|\log \varepsilon|}\right) \sim$ $|\log \varepsilon|$, and therefore estimate (1.1) is optimal in this respect ${ }^{(2)}$.

In another direction, the factor $|\log \varepsilon|$ appearing on the right-hand side of inequality (1.1) is the typical energy of a vortex solution ${ }^{(3)}$ in dimension 2 . It is known ${ }^{(4)}$ that for these solutions the integral of the potential remains bounded independently of the parameter $\varepsilon$ : Theorem 1.1 gives therefore a generalization of this fact for local integrals of the potential in arbitrary dimension, without imposing any boundary datum. More precisely, if for some constant $M_{0}>0, E_{\varepsilon}\left(u_{\varepsilon}\right) \leq$ $M_{0}|\log \varepsilon|$, then (1.1) yields the bound

$$
\int_{B_{1 / 2}} V_{\varepsilon}\left(u_{\varepsilon}\right) \leq C_{0} M_{0} \log \left(2+M_{0}\right),
$$

which is uniform in $\varepsilon$. We would also like to emphasize that Theorem 1.1 is an improvement of earlier results given in $[15,36,33,29,8]^{(5)}$.

(1) Take for instance $u_{\varepsilon}\left(x_{1}, \ldots, x_{N}\right)=\tanh \left(\frac{x_{1}}{\sqrt{2} \varepsilon}\right)$

${ }^{(2)}$ However, the optimal value of the constant $C_{0}$ is not known.

${ }^{(3)}$ A typical example is provided by a solution of the form $u_{\varepsilon}(x)=f(|x|) \exp (i \theta)$ on $B_{1} \subset \mathbb{C}$, with $f(0)=0, f(1)=1$ and $f$ verifying the ordinary differential equation $-f^{\prime \prime}+\frac{f^{\prime}}{r}=$ $\frac{1}{\varepsilon^{2}} f\left(1-f^{2}\right)$ (see e.g. [25]).

${ }^{(4)}$ This is a consequence of Pohozaev identity (see e.g. [7]).

${ }^{(5)}$ In particular, in [8] it was proved

$$
\int_{B_{1 / 2} \cap\left\{\left|u_{\varepsilon}\right| \leq 1 / 2\right\}} V_{\varepsilon}\left(u_{\varepsilon}\right) \leq C\left(\frac{E_{\varepsilon}\left(u_{\varepsilon}\right)}{|\log \varepsilon|}\right)^{2} .
$$


Remark 1.2. Using the various tools presented in this paper, one may actually derive a better estimate for the potential when the energy is small. More precisely, there exist positive constants $\eta_{1}, \beta$ and $C$ such that, if

$$
\int_{B_{1}} e_{\varepsilon}\left(u_{\varepsilon}\right) \leq \eta_{1}|\log \varepsilon|
$$

then

$$
\int_{B_{1 / 2}} V_{\varepsilon}\left(u_{\varepsilon}\right) \leq C \varepsilon^{\beta} E_{\varepsilon}\left(u_{\varepsilon}\right)
$$

so that estimate (1.1) is far from being optimal for low energy regimes. The proof of (1.3) can be obtained combining the clearing-out property (see Theorem 2.7 in Section 2) with Proposition A.4 of the Appendix. We leave it to the reader.

Using various elliptic estimates, we are able to relate, in the same spirit, local estimates for the gradient of the modulus to the potential as follows.

Proposition 1.3. Let $u_{\varepsilon}$ be a solution of $(G L)_{\varepsilon}$ on $B_{1}$. We have

$$
\int_{B_{1 / 2}}|\nabla| u_{\varepsilon}||^{2} \leq C_{1}\left(\int_{B_{1}} V_{\varepsilon}\left(u_{\varepsilon}\right)+\varepsilon\left(\int_{B_{1}} V_{\varepsilon}\left(u_{\varepsilon}\right)\right)^{1 / 2}\right)
$$

where $C_{1}$ is some constant depending only on $N$.

In contrast with Theorem 1.1, the result in Proposition 1.3 remains valid for vector-valued solutions $u_{\varepsilon}: B_{1} \rightarrow \mathbb{R}^{d}$, for arbitrary integer $d$ ( the proof carries over word for word). In the course of the proof, we invoke a bound on the $L^{4}$-norm of $\nabla u_{\varepsilon}$ (stated in Lemma 3.1), which we hope is of independent interest.

Combining Theorem 1.1 and Proposition 1.3 with covering and scaling arguments, we deduce the global bound

Corollary 1.4. Let $\Omega$ be a smooth bounded domain in $\mathbb{R}^{N}, N \geq 2$, and $u_{\varepsilon}$ be a solution of $(G L)_{\varepsilon}$. We have, for a constant $C$ depending on $\Omega$,

$$
\int_{\Omega}\left(|\nabla| u_{\varepsilon}||^{2}+V_{\varepsilon}\left(u_{\varepsilon}\right)\right)|1+\log (\operatorname{dist}(x, \partial \Omega))|^{-1} d x \leq C \Lambda\left(\frac{E_{\varepsilon}\left(u_{\varepsilon}\right)}{|\log \varepsilon|}\right),
$$

where, for $t \geq 0$, we have set $\Lambda(t)=t \log (2+t)$.

As a consequence of the above analysis, we see that in some sense the main contribution to the $G L$-energy stems from the gradient of the phase, at least in the appropriate energy regime.

In a slightly different direction, a highly involved result of Bourgain, Brezis and Mironescu yields improved interior integral estimates for $\left|\nabla u_{\varepsilon}\right|^{p}$, with $p<2$ (i.e. $p$ subcritical), in dimension three and for the energy regime $E_{\varepsilon}\left(u_{\varepsilon}\right) \leq M_{0}|\log \varepsilon|$ (see [18], Theorem 8). Our purpose is to obtain a similar result in arbitrary dimension 
and for possibly larger energy regimes. Our approach is of somewhat different nature and relies heavily on results in harmonic analysis which state that measures with suitable growth over all balls are elements of the dual of $W^{1, p}$ (see [38] and references therein).

In view of possible oscillations in the phase, which essentially propagate in the domain through an elliptic equation, it is not possible to expect a general improved estimate involving only the energy (see e.g. [20, 12]). However, these oscillations can be controlled by a weaker norm, say for instance the $L^{1}$-norm of the gradient. More precisely, we will show, as a consequence of Theorem 1.1 and [1, 2, 38], that for every $1 \leq p<2$, the following estimate holds ${ }^{(6)}$

$$
\left(\int_{B_{1 / 2}}\left|\nabla u_{\varepsilon}\right|^{p}\right)^{1 / p} \leq C_{p}\left(\Lambda\left(\frac{E_{\varepsilon}\left(u_{\varepsilon}\right)}{|\log \varepsilon|}\right)+\int_{B_{1}}\left|\nabla u_{\varepsilon}\right|\right) .
$$

Estimate (1.6) can ever be slightly improved if instead of Theorem 1.1 we invoke Jacobian estimates introduced by Jerrard and Soner [28] and Alberti, Baldo and the second author [3]. Relying on our result in [13], and the aforementioned results in [38], we obtain

Theorem 1.5. Let $1<p<2$. There exist $C_{p}>0$ such that if $u_{\varepsilon}$ is a solution to $(G L)_{\varepsilon}$, then

$$
\left(\int_{B_{1 / 2}}\left|\nabla u_{\varepsilon}\right|^{p}\right)^{1 / p} \leq C_{p}\left(\frac{E_{\varepsilon}\left(u_{\varepsilon}\right)}{|\log \varepsilon|}+\int_{B_{1}}\left|\nabla u_{\varepsilon}\right|\right) .
$$

Remark 1.6. For boundary value problems, the term $\left\|\nabla u_{\varepsilon}\right\|_{L^{1}}$ can be controlled by the trace on $\partial \Omega$. More precisely, it has been shown in $[7,29,8,18,5,9,3]$ that global bounds for $\left\|\nabla u_{\varepsilon}\right\|_{L^{p}(\Omega)}$ with $p<\frac{N}{N-1}$ could be obtained under various restrictive assumptions on $g_{\varepsilon}=u_{\varepsilon \mid \partial \Omega}$ and $u_{\varepsilon}$. The case $g_{\varepsilon}$ belonging to $H^{1 / 2}$ with the additional assumption ${ }^{(7)}\left|g_{\varepsilon}\right|=1$ was considered in [17] and then in [5,9]. The case $p=\frac{N}{N-1}$ was settled in [18] assuming a conjecture which we proved in [13] ${ }^{(8)}$.

More precisely, the results in $[3,18,13]$ yield, for $\left|g_{\varepsilon}\right|=1$ and $\Omega$ smooth bounded and simply connected ${ }^{(9)}$,

$$
\left(\int_{\Omega}\left|\nabla u_{\varepsilon}\right|^{\frac{N}{N-1}}\right)^{\frac{N-1}{N}} \leq C\left(\frac{E_{\varepsilon}\left(u_{\varepsilon}\right)}{|\log \varepsilon|}+\left\|g_{\varepsilon}\right\|_{H^{1 / 2}}^{2}\right) .
$$

${ }^{(6)}$ Inequality (1.6) yields a nontrivial result only if $E_{\varepsilon}\left(u_{\varepsilon}\right) \ll \frac{|\log \varepsilon|^{2}}{|\log | \log \varepsilon \|^{2}}$.

${ }^{(7)}$ The proof in [5] actually carries over to the case $\left|g_{\varepsilon}\right|$ is bounded away from zero, i.e. $\beta>$ $\left|g_{\varepsilon}\right|>\alpha>0$.

${ }^{(8)}$ Using a new linear estimate given in [18], Proposition 4, see also [37, 16].

(9) In [9], the assumption $\left|g_{\varepsilon}\right|=1$ is replaced by an assumption on the $G L$-energy on the boundary. 
Notice however that in the global estimate (1.8) and the case $g_{\varepsilon} \in H^{1 / 2}$ the exponent $\frac{N}{N-1}$ is the best possible (see [18]), in contrast with the result of Theorem 1.5.

Combining (1.8) and Theorem 1.5 one obtains the announced generalization of [18], Theorem 8 .

Going back to Theorem 1.5, notice that estimate (1.7) is immediate if $E_{\varepsilon}\left(u_{\varepsilon}\right) \geq$ $|\log \varepsilon|^{2}$, since by Hölder's inequality,

$$
\left(\int_{\Omega}\left|\nabla u_{\varepsilon}\right|^{p}\right)^{1 / p} \leq C\left(\int_{\Omega}\left|\nabla u_{\varepsilon}\right|^{2}\right)^{1 / 2} \leq C E_{\varepsilon}\left(u_{\varepsilon}\right)^{1 / 2} .
$$

In view of Theorem 1.5 as well as the mentioned Jacobian estimates, it is tempting to believe that estimate (1.1) can also be improved replacing possibly $\Lambda\left(\frac{E_{\varepsilon}\left(u_{\varepsilon}\right)}{|\log \varepsilon|}\right)$ by $\frac{E_{\varepsilon}\left(u_{\varepsilon}\right)}{|\log \varepsilon|}$ for suitable energy ranges ${ }^{(10)}$. In another direction, it would be interesting to extend our estimates to boundary value problems (see e.g. [22]) and to the corresponding parabolic equation.

ACKnowledgements. The third author wishes to thank warmly the Centro di Ricerca Matematica Ennio De Giorgi of the Scuola Normale Superiore di Pisa for its kind hospitality during the preparation of this work.

\section{Estimates for the potential $V_{\varepsilon}\left(u_{\varepsilon}\right)$}

The purpose of this section is to provide the proof of Theorem 1.1. The vorticity set

$$
\mathcal{V}_{\varepsilon}=\left\{x \in \Omega, \quad\left|u_{\varepsilon}\right| \leq 1-\sigma_{0}\right\},
$$

enters in an essential way in our discussion. The constant $0<\sigma_{0} \leq 1 / 2$ appearing in the definition of $\mathcal{V}_{\varepsilon}$ depends only on the dimension $N$, and will be defined at a later stage of our analysis (see Appendix).

The main point in the proof is to provide integral estimates of the potential $V_{\varepsilon}\left(u_{\varepsilon}\right)$ on small balls $B(x, r)$, distinguishing carefully the case where the balls are included in $\Omega \backslash \mathcal{V}_{\varepsilon}$ from the case where they intersect the vorticity set $\mathcal{V}_{\varepsilon}$.

\subsection{Potential estimates off the vorticity set}

We first have:

Theorem 2.1. Let $x \in \Omega$ and $r>0$ such that $B(x, r) \subset \Omega \backslash \mathcal{V}_{\varepsilon}$. There are some constants $0<\alpha_{0}<1$ and $0 \leq \sigma_{0} \leq 1 / 2$ such that if $r \geq \varepsilon$ and

$$
\tilde{E}_{\varepsilon}\left(u_{\varepsilon}, x, r\right) \equiv \frac{1}{r^{N-2}} \int_{B(x, r)} e_{\varepsilon}\left(u_{\varepsilon}\right) \leq\left(\frac{\varepsilon}{r}\right)^{-\alpha_{0}},
$$

${ }^{(10)}$ For instance, for $E_{\varepsilon}\left(u_{\varepsilon}\right) \leq \varepsilon^{-\gamma}$, for a given $0<\gamma<1$. 
then

$$
\left|u_{\varepsilon}\right| \geq 1-\frac{C \varepsilon^{2}}{r^{2}}\left(1+\tilde{E}_{\varepsilon}\left(u_{\varepsilon}, x, r\right)\right) \quad \text { on } \quad B(x, r / 2) .
$$

The proof of Theorem 2.1 follows some arguments in [11, 14]. The necessary adaptation will be exposed in a separate Appendix. Theorem 2.1 provides a lower bound for $\left|u_{\varepsilon}\right|$ off the vorticity set. On the other hand, we recall some important well-known global upper bounds.

Proposition 2.2. Let $\Omega$ be a smooth domain in $\mathbb{R}^{N}$ and $u_{\varepsilon}$ be a solution of $(G L)_{\varepsilon}$ on $\Omega$. There exists a constant $C>0$ depending only on $N$ such that, if $\operatorname{dist}(x, \partial \Omega)>$ $\sqrt{\varepsilon}$, then

$$
\begin{gathered}
\left|u_{\varepsilon}(x)\right| \leq 1+\frac{C \varepsilon^{2}}{\operatorname{dist}(x, \partial \Omega)}, \\
\left|\nabla u_{\varepsilon}(x)\right| \leq \frac{C}{\varepsilon} .
\end{gathered}
$$

Combining Proposition 2.2 and Theorem 2.1, we deduce immediately the following uniform estimate for the potential on balls included in $\Omega \backslash \mathcal{V}_{\varepsilon}$.

Proposition 2.3. Let $x, r$ and $u_{\varepsilon}$ be as in Theorem 2.1 and assume that $r \geq \varepsilon$ and $\operatorname{dist}(x, \partial \Omega)>\sqrt{\varepsilon}$. Then

$$
\sup _{B(x, r / 2)} V_{\varepsilon}\left(u_{\varepsilon}\right) \leq C \frac{\varepsilon^{2}}{r^{4}}\left(1+\tilde{E}_{\varepsilon}\left(u_{\varepsilon}, x, r\right)\right)^{2} .
$$

In the proof of Theorem 1.1 we will invoke Proposition 2.3 for balls of fixed radius $r=r_{0}$, specifying throughout the choice of $r_{0}$ in a somewhat arbitrary way as

$$
r_{0}=\frac{\varepsilon^{1 / 4}}{2}
$$

For this choice of $r_{0}$, the factor $\frac{\varepsilon^{2}}{r_{0}^{4}}$ is equal to $16 \varepsilon$, and hence small.

\subsection{Potential estimates on the vorticity set}

In this section, we consider the case where $B\left(x, r_{0}\right)$ intersects the vorticity set $\mathcal{V}_{\varepsilon}$, that is

$$
\operatorname{dist}\left(x, \mathcal{V}_{\varepsilon}\right) \leq \frac{\varepsilon^{1 / 4}}{2}
$$

In this situation, we show 
Proposition 2.4. Assume that dist $(x, \partial \Omega) \geq \varepsilon^{1 / 8}$ and that (2.6) holds. Then there exists some radius $\varepsilon^{1 / 4} \leq r(x) \leq \varepsilon^{1 / 8}$ depending only on $u_{\varepsilon}$ and $x$ such that

$$
\int_{B(x, r(x))} V_{\varepsilon}\left(u_{\varepsilon}\right) \leq \frac{C}{|\log \varepsilon|} \log \left(2+\frac{\tilde{E}_{\varepsilon}\left(x, u_{\varepsilon}, \varepsilon^{1 / 8}\right)}{|\log \varepsilon|}\right) \int_{B(x, r(x))} e_{\varepsilon}\left(u_{\varepsilon}\right),
$$

where $C>0$ is some constant depending only on $N$.

Comment. 1) We would like to draw the attention of the reader to the fact that the 1.h.s. of (2.7) is the potential integrated on the same domain $B(x, r(x))$ as the energy $e_{\varepsilon}\left(u_{\varepsilon}\right)$ on the right-hand side of the inequality. However the scaled energy $\tilde{E}_{\varepsilon}\left(x, u_{\varepsilon}, \varepsilon^{1 / 8}\right)$ is considered on the larger domain $B\left(x, \varepsilon^{1 / 8}\right)$.

2) In contrast with Proposition 2.3, the radius $r(x)$ for which this inequality holds is not fixed a priori, but depends on $x$.

The main tools in the proof are the monotonicity formula and the clearing-out theorem which we recall first.

Proposition 2.5 (Monotonicity formula $\left.{ }^{(11)}\right)$. Assume $u_{\varepsilon}$ is a solution of $(G L)_{\varepsilon}$ in $B(x, R)$. Then we have

$$
\begin{gathered}
\frac{d}{d r}\left(\tilde{E}_{\varepsilon}\left(u_{\varepsilon}, x, r\right)\right)=\frac{1}{r^{N-2}} \int_{\partial B(x, r)}\left|\frac{\partial u_{\varepsilon}}{\partial n}\right|^{2}+\frac{1}{r^{N-1}} \int_{B(x, r)} \frac{\left(1-\left|u_{\varepsilon}\right|^{2}\right)^{2}}{2 \varepsilon^{2}}, \\
\text { for } 0<r<R
\end{gathered}
$$

Remark 2.6. Note in particular that

$$
\frac{d}{d r}\left(\tilde{E}_{\varepsilon}\left(u_{\varepsilon}, x, r\right)\right) \geq \frac{1}{r^{N-1}} \int_{B(x, r)} \frac{\left(1-\left|u_{\varepsilon}\right|^{2}\right)^{2}}{2 \varepsilon^{2}} \geq 0,
$$

so that for fixed $x$, the function $F(r) \equiv \tilde{E}_{\varepsilon}\left(u_{\varepsilon}, x, r\right)$ is a nondecreasing function of the radius $r$. Moreover, setting

$$
G(r)=r^{2-N} \int_{B(x, r)} \frac{\left(1-\left|u_{\varepsilon}\right|^{2}\right)^{2}}{2 \varepsilon^{2}},
$$

(2.8) yields the differential inequality

$$
r F^{\prime} \geq G
$$

which relates in a simple way the energy integral and the potential integral on $B(x, r)$. This relation will be exploited in the proof of Proposition 2.4.

${ }^{(11)}$ Here we consider the version given in [8]. Earlier versions were given in [36, 33, 29]. 
Theorem 2.7 (Clearing-out ${ }^{(12)}$ for vorticity). Let $u_{\varepsilon}$ be a solution to $(G L)_{\varepsilon}, x \in \Omega$, $r \geq \varepsilon$ such that $B(x, r) \subset \Omega$. If

$$
\mathcal{V}_{\varepsilon} \cap B\left(x, \frac{r}{2}\right) \neq \emptyset,
$$

then

$$
\tilde{E}_{\varepsilon}\left(u_{\varepsilon}, x, r\right) \geq \eta_{0}\left|\log \left(\frac{\varepsilon}{r}\right)\right|,
$$

where $\eta_{0}>0$ is some constant depending only on $N$.

Proof of Proposition 2.4. For $s \in I_{\varepsilon} \equiv\left[\frac{1}{4} \log \varepsilon, \frac{1}{8} \log \varepsilon\right]$, set $f(s)=F(\exp s)$, $g(s)=G(\exp s)$, so that relation (2.9) writes, for $f$ and $g$,

$$
f^{\prime}(s) \geq g(s) \quad \forall s \in I_{\varepsilon} .
$$

For this ODE we invoke the next elementary lemma.

Lemma 2.8. Let $I=[a, b]$ be a bounded interval of $\mathbb{R}, f$ and $g$ two nonnegative absolutely continuous functions such that

$$
f^{\prime} \geq g \quad \text { on } \quad I \text {. }
$$

Then there exists $s_{0} \in I$ such that

$$
g\left(s_{0}\right) \leq \frac{1}{b-a} \log \left(\frac{f(b)}{f(a)}\right) f\left(s_{0}\right) .
$$

Applying Lemma 2.8 to (2.10) we deduce that there exists some $r(x) \in\left[\varepsilon^{1 / 4}, \varepsilon^{1 / 8}\right]$ such that

$$
G(r(x)) \leq \frac{1}{|\log \varepsilon|} \log \left(\frac{F\left(\varepsilon^{1 / 8}\right)}{F\left(\varepsilon^{1 / 4}\right)}\right) F(r(x))
$$

On the other hand, by the clearing-out theorem, since $B\left(x, \frac{\varepsilon^{1 / 4}}{2}\right) \cap \mathcal{V}_{\varepsilon} \neq \emptyset$, we have

$$
F\left(\varepsilon^{1 / 4}\right)=\tilde{E}_{\varepsilon}\left(x, u_{\varepsilon}, \varepsilon^{1 / 4}\right) \geq \eta_{0}\left|\log \left(\frac{\varepsilon}{\varepsilon^{1 / 4}}\right)\right|=\frac{3 \eta_{0}}{4}|\log \varepsilon| .
$$

Combining (2.12) and (2.13), we deduce the conclusion (2.7).

Proof of Lemma 2.8 The argument is by contradiction. If (2.11) were false, we would have

$$
f^{\prime}(s)>\lambda f(s) \quad \forall s \in[a, b],
$$

${ }^{(12)}$ This kind of result was proved under various assumptions in $[15,36,33,29,30,8,10]$ and termed $\eta$-compactness in $[33,29,30], \eta$-ellipticity in $[8,10]$. Here we follow the terminology in [27, 11], which was introduced by Brakke [19]. 
where we have set

$$
\lambda=\frac{1}{b-a} \log \left(\frac{f(b)}{f(a)}\right)
$$

so that

$$
\frac{d}{d s}(\exp (-\lambda s) f(s))>0 \quad \text { on } \quad[a, b] .
$$

Hence, by integration

$$
f(b)>\exp (-\lambda(a-b)) f(a)=f(b),
$$

a contradiction.

\subsection{Proof of Theorem 1.1}

Recall that in this section $\Omega \equiv B_{1}$. First, notice that we may assume throughout that $u_{\varepsilon}$ satisfies the energy bound

$$
E_{\varepsilon}\left(u_{\varepsilon}\right) \leq \varepsilon^{-\beta_{0}}
$$

where $\beta_{0}=\frac{\alpha_{0}}{2}, \alpha_{0}$ being the constant appearing in Theorem A.2. Indeed, if otherwise $E_{\varepsilon}\left(u_{\varepsilon}\right) \geq \varepsilon^{-\beta_{0}}$, then, as already mentioned in the introduction, the conclusion is straightforward, choosing $C_{0}=\frac{2}{\beta_{0}}$.

We next consider the sets

$$
\Lambda_{\varepsilon}=\left\{x \in B_{1 / 2}, \operatorname{dist}\left(x, \mathcal{V}_{\varepsilon}\right) \leq r_{0}=\frac{\varepsilon^{1 / 4}}{2}\right\} \quad \text { and } \quad \Gamma_{\varepsilon}=B_{1 / 2} \backslash \Lambda_{\varepsilon},
$$

so that $\Lambda_{\varepsilon} \cup \Gamma_{\varepsilon}=B_{1 / 2}$.

Step 1: bounds for the potential on $\Gamma_{\varepsilon}$. If $x \in \Gamma_{\varepsilon}$, then $B\left(x, r_{0}\right) \subset B_{3 / 4} \backslash \mathcal{V}_{\varepsilon}$ provided $\varepsilon$ is sufficiently small, and we may therefore invoke Proposition 2.3 to assert that

$$
V_{\varepsilon}\left(u_{\varepsilon}(x)\right) \leq C \varepsilon\left(1+\tilde{E}_{\varepsilon}\left(u_{\varepsilon}, x, r_{0}\right)\right)^{2}
$$

By monotonicity we have

$$
\tilde{E}_{\varepsilon}\left(u_{\varepsilon}, x, r_{0}\right) \leq \tilde{E}_{\varepsilon}\left(u_{\varepsilon}, x, r_{0}+\frac{1}{4}\right) \leq C E_{\varepsilon}\left(u_{\varepsilon}\right) .
$$

Combining (2.15) with (2.16), together with the fact that $E_{\varepsilon}\left(u_{\varepsilon}\right) \leq \varepsilon^{-\beta} \leq \varepsilon^{-1 / 2}$, we deduce

$$
\sup _{\Gamma_{\varepsilon}} V_{\varepsilon}\left(u_{\varepsilon}\right) \leq C \varepsilon^{1 / 2}\left(1+E_{\varepsilon}\left(u_{\varepsilon}\right)\right)
$$


Step 2: integrating the potential on $\Lambda_{\varepsilon}$. If $x \in \Lambda_{\varepsilon}$, then dist $\left(x, \mathcal{V}_{\varepsilon}\right) \leq r_{0}$, so that we may apply Proposition 2.4. Combining (2.7) with the monotonicity formula as above, we deduce

$$
\int_{B(x, r(x))} V_{\varepsilon}\left(u_{\varepsilon}\right) \leq \frac{C}{|\log \varepsilon|} \int_{B(x, r(x))} e_{\varepsilon}\left(u_{\varepsilon}\right) \log \left(2+\frac{E_{\varepsilon}\left(u_{\varepsilon}\right)}{|\log \varepsilon|}\right) .
$$

We next consider the covering $\cup_{x \in \Lambda_{\varepsilon}} B(x, r(x))$ of $\Lambda_{\varepsilon}$ and apply Besicovitch covering Theorem. This yields a finite set $A=\left\{x_{1}, \ldots, x_{m}\right\} \subset \Lambda_{\varepsilon}$ such that

$$
\Lambda_{\varepsilon} \subset \cup_{x \in A} B(x, r(x))
$$

and the balls $B\left(x_{i}, r\left(x_{i}\right)\right), i=1, \ldots, m$ can be distributed in $\ell$ families $\mathcal{B}_{k}, k=$ $1, \ldots, \ell$ of disjoint closed balls, where $\ell$ is a constant depending only on $N$. We write

$$
\begin{aligned}
\int_{\Lambda_{\varepsilon}} V_{\varepsilon}\left(u_{\varepsilon}\right) & \leq \sum_{i=1}^{m} \int_{B\left(x_{i}, r\left(x_{i}\right)\right)} V_{\varepsilon}\left(u_{\varepsilon}\right) \\
& \leq \frac{C}{|\log \varepsilon|} \log \left(2+\frac{E_{\varepsilon}\left(u_{\varepsilon}\right)}{|\log \varepsilon|}\right) \sum_{i=1}^{m} \int_{B\left(x_{i}, r\left(x_{i}\right)\right)} e_{\varepsilon}\left(u_{\varepsilon}\right)
\end{aligned}
$$

On the other hand,

$$
\begin{aligned}
\sum_{i=1}^{m} \int_{B\left(x_{i}, r\left(x_{i}\right)\right)} e_{\varepsilon}\left(u_{\varepsilon}\right) & \leq \sum_{k=1}^{\ell}\left(\sum_{B\left(x_{i}, r\left(x_{i}\right)\right) \in \mathcal{B}_{k}} \int_{B\left(x_{i}, r\left(x_{i}\right)\right)} e_{\varepsilon}\left(u_{\varepsilon}\right)\right) \\
& \leq \ell E_{\varepsilon}\left(u_{\varepsilon}\right) .
\end{aligned}
$$

Combining (2.18), (2.19) and (2.20), we obtain

$$
\int_{\Lambda_{\varepsilon}} V_{\varepsilon}\left(u_{\varepsilon}\right) \leq \frac{C}{|\log \varepsilon|} E_{\varepsilon}\left(u_{\varepsilon}\right) \log \left(2+\frac{E_{\varepsilon}\left(u_{\varepsilon}\right)}{|\log \varepsilon|}\right) .
$$

Step 3: Proof of Theorem 1.1 completed. We distinguish two cases.

Case A: $E_{\varepsilon}\left(u_{\varepsilon}\right) \geq \varepsilon^{1 / 2}|\log \varepsilon|$. It follows from (2.17) that

$$
\sup _{\Gamma_{\varepsilon}} V_{\varepsilon}\left(u_{\varepsilon}\right) \leq C \frac{E_{\varepsilon}\left(u_{\varepsilon}\right)}{|\log \varepsilon|}
$$

so that the conclusion (1.1) follows from (2.22) and (2.21).

Case B: $E_{\varepsilon}\left(u_{\varepsilon}\right) \leq \varepsilon^{1 / 2}|\log \varepsilon|$. In this case it follows from the clearing-out theorem that if $\varepsilon$ is sufficiently small,

$$
\left|u_{\varepsilon}\right| \geq 1-\sigma_{0} \quad \text { on } \quad B_{9 / 10}
$$

so that $\Lambda_{\varepsilon}=\emptyset$, and $\Gamma_{\varepsilon}=B_{1 / 2}$. The conclusion (1.1) then follows from Proposition A. 4 of the Appendix. 
Remark 2.9. Let $\varepsilon<r<1$. The analogue of (1.1) for the ball $B_{r}$ writes, by scaling,

$$
\int_{B_{r / 2}} V_{\varepsilon}\left(u_{\varepsilon}\right) \leq \frac{E_{\varepsilon}\left(u_{\varepsilon}, r\right)}{\left|\log \frac{\varepsilon}{r}\right|} \log \left(2+\frac{E_{\varepsilon}\left(u_{\varepsilon}, r\right)}{r^{N-2}\left|\log \frac{\varepsilon}{r}\right|}\right),
$$

where $E_{\varepsilon}\left(u_{\varepsilon}, r\right) \equiv \int_{B_{r}} e_{\varepsilon}\left(u_{\varepsilon}\right)=r^{N-2} \tilde{E}_{\varepsilon}\left(u_{\varepsilon}, 0, r\right)$. Indeed, setting $\tilde{u}_{\varepsilon}(\tilde{x})=u_{\varepsilon}(r \tilde{x})$ for $\tilde{x} \in B_{1}$, we verify that $\tilde{u}_{\varepsilon}$ is a solution of (GL) $\tilde{\varepsilon}$ on $B_{1}$, for $\tilde{\varepsilon}=\varepsilon / r$. Moreover, we have

$$
E_{\varepsilon}\left(\tilde{u}_{\varepsilon}\right)=\frac{1}{r^{N-2}} E_{\varepsilon}\left(u_{\varepsilon}, r\right), \quad \int_{B_{1}} V_{\tilde{\varepsilon}}\left(\tilde{u}_{\tilde{\varepsilon}}\right)=\frac{1}{r^{N-2}} \int_{B_{r}} V_{\varepsilon}\left(u_{\varepsilon}\right) .
$$

\section{Gradient estimates for the modulus $\left|u_{\varepsilon}\right|$}

The aim of this section is to prove Proposition 1.3. The starting point is the elliptic equation for the function $\zeta \equiv 1-\rho_{\varepsilon}^{2} \equiv 1-\left|u_{\varepsilon}\right|^{2}$,

$$
-\Delta \zeta+2 \frac{\rho_{\varepsilon}^{2}}{\varepsilon^{2}} \zeta=2\left|\nabla u_{\varepsilon}\right|^{2}
$$

which is a straightforward consequence of $(\mathrm{GL})_{\varepsilon}$. We introduce next a test function $\chi \in \mathcal{C}_{c}^{\infty}\left(B_{1}\right)$ such that $\chi \equiv 1$ on $B_{1 / 2}$ and $\chi=0$ on $B_{1} \backslash B_{3 / 4}$. Multiplying (3.1) by $\chi^{2} \zeta$ and integrating by parts, we obtain, after a few standard computations,

$$
\frac{1}{2} \int_{B_{1 / 2}}|\nabla \zeta|^{2} \leq 2 \int_{B_{3 / 4}} \zeta\left|\nabla u_{\varepsilon}\right|^{2}+2 \int_{B_{3 / 4}} \zeta^{2}|\nabla \chi|^{2}
$$

On the other hand, we have the pointwise equality $|\nabla \zeta|^{2}=\left.\left.|\nabla| u_{\varepsilon}\right|^{2}\right|^{2}=4\left|u_{\varepsilon}\right|^{2}|\nabla| u_{\varepsilon}||^{2}$, so that

$$
|\nabla| u_{\varepsilon}||^{2}=\frac{1}{4}|\nabla \zeta|^{2}+\left(1-\left|u_{\varepsilon}\right|^{2}\right)|\nabla| u_{\varepsilon}||^{2} \leq \frac{1}{4}|\nabla \zeta|^{2}+|\zeta||\nabla| u_{\varepsilon}||^{2},
$$

and hence (3.2) yields

$$
\int_{B_{1 / 2}}|\nabla| u_{\varepsilon}||^{2} \leq 2 \frac{3}{2} \int_{B_{3 / 4}}|\zeta|\left|\nabla u_{\varepsilon}\right|^{2}+\int_{B_{3} / 4} \zeta^{2}|\nabla \chi|^{2} .
$$

To complete the proof of Proposition 1.3, we apply Cauchy-Schwarz inequality to the first integral on the right-hand side of (3.3). This involves the term $\left\|\nabla u_{\varepsilon}\right\|_{L^{4}\left(B_{3 / 4}\right)}$, which is handled by the following

Lemma 3.1. We have

$$
\int_{B_{3 / 4}}\left|\nabla u_{\varepsilon}\right|^{4} \leq C\left(1+\frac{1}{\varepsilon^{2}} \int_{B_{4 / 5}} V_{\varepsilon}\left(u_{\varepsilon}\right)\right),
$$

where $C>0$ is a constant depending only possibly on $N$. 
Comment. The factor $\varepsilon^{-2}$ in (3.4) is somewhat optimal. This can be checked both for vortices and kinks. For vortices, i.e. planar complex solutions of the form $u_{\varepsilon}=f_{\varepsilon}(r) \exp (i \theta)$, we have

$$
\int_{B_{3 / 4}}\left|\nabla u_{\varepsilon}\right|^{4} \simeq C \int_{\varepsilon}^{3 / 4} \frac{1}{r^{4}} r d r \simeq \frac{C}{\varepsilon^{2}} \simeq \frac{C}{\varepsilon^{2}} \int_{B_{1}} V_{\varepsilon}\left(u_{\varepsilon}\right),
$$

whereas for the standard kink $\tanh \left(\frac{x}{\sqrt{2} \varepsilon}\right)$ we have

$$
\int_{B_{3 / 4}}\left|\nabla u_{\varepsilon}\right|^{4} \simeq C \int_{-\varepsilon}^{\varepsilon} \frac{1}{\varepsilon^{4}} d x \simeq \frac{C}{\varepsilon^{3}} \simeq \frac{C}{\varepsilon^{2}} \int_{B_{1}} V_{\varepsilon}\left(u_{\varepsilon}\right) .
$$

Proof of Lemma 3.1 On the ball $B_{4 / 5}$ we decompose the function $u_{\varepsilon}$ as $u_{\varepsilon} \equiv v_{\varepsilon}+w_{\varepsilon}$, where $w_{\varepsilon}$ is a harmonic function and verifies $w_{\varepsilon}=u_{\varepsilon}$ on the boundary $\partial B_{4 / 5}$. In view of Proposition 2.2, $u_{\varepsilon}$, and hence $w_{\varepsilon}$ are uniformly bounded on $\partial B_{4 / 5}$, so that, since $w_{\varepsilon}$ is harmonic,

$$
\left|\nabla w_{\varepsilon}\right| \leq C \quad \text { on } \quad B_{3 / 4},
$$

where $C$ depends only on the dimension $N$.

Turning to $v_{\varepsilon}$, we notice that by construction $v_{\varepsilon}=0$ on $\partial B_{4 / 5}$, and that

$$
\left|\Delta v_{\varepsilon}\right| \leq \frac{C}{\varepsilon} V_{\varepsilon}\left(u_{\varepsilon}\right)^{1 / 2} .
$$

Hence by standard elliptic theory

$$
\left\|v_{\varepsilon}\right\|_{H^{2}\left(B_{4 / 5}\right)}^{2} \leq \frac{C}{\varepsilon^{2}} \int_{B_{4 / 5}} V_{\varepsilon}\left(u_{\varepsilon}\right) .
$$

Moreover, since $u_{\varepsilon}$ and $w_{\varepsilon}$ are uniformly bounded on $B_{4 / 5}$, the same holds for $v_{\varepsilon}$, i.e.

$$
\left\|v_{\mathcal{E}}\right\|_{L^{\infty}\left(B_{4 / 5}\right)} \leq C,
$$

where $C>0$ is depending only on $N$. We next invoke a classical GagliardoNirenberg type inequality which asserts that

$$
\left\|\nabla v_{\varepsilon}\right\|_{L^{4}\left(B_{4 / 5}\right)}^{2} \leq C|| v_{\varepsilon}\left\|_{H^{2}}\right\| v_{\varepsilon} \|_{L^{\infty}},
$$

where $C$ depends only on $N$. An elementary proof of (3.8) may actually be derived as follows. Since $v \equiv v_{\varepsilon}$ is compactly supported in $B_{4 / 5}$, we may write, for $i=$ $1, \ldots, N$, integrating by parts,

$$
\begin{aligned}
\left\|v_{x_{i}}\right\|_{L^{4}\left(B_{4 / 5}\right)}^{4}=\int v_{x_{i}}^{3} v_{x_{i}} & =-\int\left(v_{x_{i}}^{3}\right)_{x_{i}} v=-3 \int v_{x_{i} x_{i}}\left(v_{x_{i}}\right)^{2} v \\
& \leq 3\left\|v_{x_{i} x_{i}}\right\|_{L^{2}}\left\|v_{x_{i}}\right\|_{L^{4}}^{2}\|v\|_{L^{\infty}}
\end{aligned}
$$


which yields (3.8). Combining (3.6), (3.7) and (3.8), we obtain

$$
\left\|\nabla v_{\varepsilon}\right\|_{L^{4}\left(B_{4 / 5}\right)}^{4} \leq \frac{C}{\varepsilon^{2}} \int_{B_{4 / 5}} V_{\varepsilon}\left(u_{\varepsilon}\right) .
$$

Invoking finally (3.5) together with the decomposition $u_{\varepsilon}=v_{\varepsilon}+w_{\varepsilon}$ we complete the proof of Lemma 3.1

Remark 3.2. In view of the inequality $\left|\nabla u_{\varepsilon}\right| \leq C / \varepsilon$, we deduce by (3.5) that $\left|\nabla v_{\varepsilon}\right| \leq C / \varepsilon$ on $B_{3 / 4}$. It follows that for any $p \geq 4$ we have

$$
\left\|\nabla v_{\varepsilon}\right\|_{L^{p}\left(B_{3 / 4}\right)}^{p} \leq \frac{C_{p}}{\varepsilon^{p-2}} \int_{B_{4 / 5}} V_{\varepsilon}\left(u_{\varepsilon}\right),
$$

and hence, for every $p \geq 4$,

$$
\int_{B_{3 / 4}}\left|\nabla u_{\varepsilon}\right|^{p} \leq C_{p}\left(\frac{1}{\varepsilon^{p-2}} \int_{B_{4 / 5}} V_{\varepsilon}\left(u_{\varepsilon}\right)+1\right) .
$$

As in the case $p=4$, the exponent $\varepsilon^{-(p-2)}$ is somewhat optimal. We conjecture actually that inequality (3.11) is true for every $p>2$.

Proof of Proposition 1.3 completed. We go back to inequality (3.3). In view of Lemma 3.1, we have, by Cauchy-Schwarz inequality

$$
\begin{aligned}
\int_{B_{3 / 4}}|\zeta|\left|\nabla u_{\varepsilon}\right|^{2} & \leq\|\zeta\|_{L^{2}\left(B_{3 / 4}\right)}|| \nabla u_{\varepsilon} \|_{L^{4}\left(B_{3 / 4}\right)}^{2} \\
& \leq C\left(\int_{B_{3 / 4}} \varepsilon^{2} V_{\varepsilon}\left(u_{\varepsilon}\right)\right)^{1 / 2}\left(1+\int_{B_{4 / 5}} \varepsilon^{-2} V_{\varepsilon}\left(u_{\varepsilon}\right)\right)^{1 / 2} \\
& \leq C\left(\int_{B_{4 / 5}} V_{\varepsilon}\left(u_{\varepsilon}\right)+\varepsilon\left(\int_{B_{4 / 5}} V_{\varepsilon}\left(u_{\varepsilon}\right)\right)^{1 / 2}\right)
\end{aligned}
$$

The conclusion follows.

Remark 3.3. Combining Theorem 1.1 and Proposition 1.3 we are immediately led to the inequality

$$
\int_{B_{1 / 2}}|\nabla| u_{\varepsilon}||^{2} \leq C_{2} \Lambda\left(\frac{E_{\varepsilon}\left(u_{\varepsilon}\right)}{|\log \varepsilon|}\right) .
$$

Indeed, as in the proof of Theorem 1.1 one distinguishes two cases:

Case 1: $\int_{B_{4 / 5}} V_{\varepsilon}\left(u_{\varepsilon}\right) \geq \varepsilon^{2}$. In this case $\varepsilon\left(\int_{B_{4 / 5}} V_{\varepsilon}\left(u_{\varepsilon}\right)\right)^{1 / 2} \leq \int_{B_{4 / 5}} V_{\varepsilon}\left(u_{\varepsilon}\right)$ and the conclusion follows directly from (1.4).

Case 2: $\int_{B_{4 / 5}} V_{\varepsilon}\left(u_{\varepsilon}\right) \leq \varepsilon^{2}$. One concludes in this case using Proposition A.4. 
Remark 3.4. As in Remark 2.9 we may argue by scaling to assert that, for $\varepsilon<r<1$, we have

$$
\int_{B_{r / 2}}|\nabla| u_{\varepsilon}||^{2}+V_{\varepsilon}\left(u_{\varepsilon}\right) \leq \frac{E_{\varepsilon}\left(u_{\varepsilon}, r\right)}{\left|\log \frac{\varepsilon}{r}\right|} \log \left(2+\frac{E_{\varepsilon}\left(u_{\varepsilon}, r\right)}{r^{N-2}\left|\log \frac{\varepsilon}{r}\right|}\right) .
$$

Proof of Corollary 1.4. Let $\Omega$ be an arbitrary smooth domain in $\mathbb{R}^{N}$. Let $\varepsilon^{1 / 2} \leq$ $r \leq 1$ and consider a ball $B(x, r) \subset \Omega$. As a direct consequence of (3.14) and the elementary inequality $\log \left(2+\frac{E}{r^{N-2}}\right) \leq \log (2+E)(1+|\log r|)$, it follows that

$$
\int_{B(x, r / 2)}|\nabla| u_{\varepsilon}||^{2}+V_{\varepsilon}\left(u_{\varepsilon}\right) \leq \frac{C}{|\log \varepsilon|} \log \left(2+\frac{E_{\varepsilon}\left(u_{\varepsilon}\right)}{|\log \varepsilon|}\right)(1+|\log r|) \int_{B(x, r)} e_{\varepsilon}\left(u_{\varepsilon}\right) .
$$

Using a covering argument, we deduce

$$
\int_{\left\{\operatorname{dist}(x, \partial \Omega) \geq \varepsilon^{1 / 4}\right\}}\left(|\nabla| u_{\varepsilon}||^{2}+V_{\varepsilon}\left(u_{\varepsilon}\right)\right)|1+\log (\operatorname{dist}(x, \partial \Omega))|^{-1} d x \leq C \Lambda\left(\frac{E_{\varepsilon}\left(u_{\varepsilon}\right)}{|\log \varepsilon|}\right) .
$$

On the other hand,

$$
\begin{aligned}
& \int_{\left\{\operatorname{dist}(x, \partial \Omega) \leq \varepsilon^{1 / 4}\right\}}\left(|\nabla| u_{\varepsilon}||^{2}+V_{\varepsilon}\left(u_{\varepsilon}\right)\right)|1+\log (\operatorname{dist}(x, \partial \Omega))|^{-1} d x \\
& \leq \frac{E_{\varepsilon}\left(u_{\varepsilon}\right)}{1+\frac{1}{4}|\log \varepsilon|} \leq C \frac{E_{\varepsilon}\left(u_{\varepsilon}\right)}{|\log \varepsilon|} .
\end{aligned}
$$

Combining the last two inequalities, the conclusion (1.5) follows.

\section{4. $L^{p}$ estimates for $\nabla u_{\varepsilon}, p<2$}

The purpose of this section is to prove (1.6) and its improvement (1.7) stated in Theorem 1.5. Throughout this section, our arguments follow closely methods introduced in the afore quoted literature. We first decompose the gradient into contributions of phase and modulus, writing (here and in the sequel $u \equiv u_{\varepsilon}$ )

$$
4|u|^{2}|\nabla u|^{2}=4|u \times \nabla u|^{2}+\left.\left.|\nabla| u\right|^{2}\right|^{2},
$$

so that

$$
4|\nabla u|^{2}=4|u \times \nabla u|^{2}+\left.\left.|\nabla| u\right|^{2}\right|^{2}+4\left(1-|u|^{2}\right)|\nabla u|^{2} .
$$

For the two last terms on the right-hand side of (4.2) we invoke our previous estimate (3.13) and (3.12), so that

$$
\left.\left.\int_{B_{1 / 2}}|\nabla| u\right|^{2}\right|^{2}+\left.\left.4|1-| u\right|^{2}|| \nabla u\right|^{2} \leq C \Lambda\left(\frac{E_{\varepsilon}\left(u_{\varepsilon}\right)}{|\log \varepsilon|}\right)
$$


so that, by Hölder inequality,

$$
\left(\left.\left.\int_{B_{1 / 2}}|\nabla| u\right|^{2}\right|^{p}+4\left(\left.\left.|1-| u\right|^{2}|| \nabla u\right|^{2}\right)^{p / 2}\right)^{1 / p} \leq C\left(\Lambda\left(\frac{E_{\varepsilon}\left(u_{\varepsilon}\right)}{|\log \varepsilon|}\right)\right)^{1 / 2} .
$$

It follows in particular, that if $E_{\varepsilon}\left(u_{\varepsilon}\right) \geq \eta_{1}|\log \varepsilon|$ (where $\eta_{1}$ is the constant appearing in Theorem A.7 of the Appendix), then since $\left(\Lambda\left(\frac{E_{\varepsilon}\left(u_{\varepsilon}\right)}{|\log \varepsilon|}\right)\right)^{1 / 2} \leq C \frac{E_{\varepsilon}\left(u_{\varepsilon}\right)}{|\log \varepsilon|}$, we deduce

$$
\left(\left.\left.\int_{B_{1 / 2}}|\nabla| u\right|^{2}\right|^{p}+4\left(\left.\left.|1-| u\right|^{2}|| \nabla u\right|^{2}\right)^{p / 2}\right)^{1 / p} \leq C \frac{E_{\varepsilon}\left(u_{\varepsilon}\right)}{|\log \varepsilon|} .
$$

\subsection{Decomposing $u \times \nabla u$}

Recall that the term $u \times \nabla u$ represents essentially the gradient of the phase ${ }^{(13)}$, and verifies the equation $\operatorname{div}(u \times \nabla u)=0^{(14)}$. It is convenient to rewrite this equation in the formalism of differential forms ${ }^{(15)}$

$$
d^{*}(u \times d u)=0 .
$$

In order to derive an elliptic system for $u \times d u$ it is useful to consider the Jacobian $J u \equiv d(u \times d u)=2 \sum_{i<j} u_{x_{i}} \times u_{x_{j}} d x_{i} \wedge d x_{j}$ as well as the truncated quantity

$$
\tilde{J}=d(\chi u \times d u)=\chi J u+d \chi \wedge(u \times d u),
$$

where $0 \leq \chi \leq 1$ denotes a smooth function, compactly supported in $B_{7 / 8}$, and such that $\chi \equiv 1$ on $B_{6 / 7}$. Let $\psi$ be the solution of the elliptic equation

$$
\Delta \psi=\tilde{J} \quad \text { on } \quad \mathbb{R}^{N},
$$

obtained through convolution with the fundamental solution of the Laplacian. Since $d \tilde{J}=0$, it follows that $\Delta(d \psi)=0$, so that $d \psi=0$ on $\mathbb{R}^{N}$. Hence, we are led to

$$
d d^{*} \psi=d d^{*} \psi+d^{*} d \psi=\Delta \psi=\tilde{J}
$$

so that

$$
d\left(u \times d u-d^{*} \psi\right)=0 \quad \text { on } \quad B_{6 / 7} .
$$

By Poincaré Lemma, there exists some function $\Phi$ on $B_{6 / 7}$ such that

$$
u \times d u=d \Phi+d^{*} \psi \quad \text { on } \quad B_{6 / 7} .
$$

${ }^{(13)}$ Indeed, if $u \neq 0$, then $u=|u| \exp (i \varphi)$ and $u \times \nabla u=|u|^{2} \nabla \varphi$.

${ }^{(14)}$ Take the exterior product of $(\mathrm{GL})_{\varepsilon}$ with $u$.

${ }^{(15)} d$ denotes the exterior differential, and $d^{*}= \pm \star d \star$ its formal adjoint. 
Moreover, applying $d^{*}$ to both sides we deduce

$$
\Delta \Phi=0 \quad \text { on } \quad B_{6 / 7}
$$

By standard elliptic estimates it follows

$$
\|\nabla \Phi\|_{L^{\infty}\left(B_{5 / 6}\right)} \leq C\|\nabla u\|_{L^{1}\left(B_{6 / 7}\right)}+\|\nabla \psi\|_{L^{1}\left(B_{6 / 7}\right)} .
$$

Next, we write $\psi=\psi_{1}+\psi_{2}$, where $\psi_{2}$ is the solution (obtained through convolution with the fundamental solution of the Laplacian) of the equation

$$
\Delta \psi_{2}=d \chi \wedge(u \times d u) \quad \text { on } \mathbb{R}^{N} .
$$

Clearly, by standard elliptic estimates,

$$
\left\|\nabla \psi_{2}\right\|_{L^{\infty}\left(B_{7 / 8}\right)} \leq C\|\nabla u\|_{L^{1}\left(B_{8 / 9}\right)} .
$$

At this stage it remains to analyze the term $\psi_{1}$, solution of

$$
\Delta \psi_{1}=\chi J u \quad \text { on } \quad \mathbb{R}^{N},
$$

which is the central part of the proofs of inequalities (1.6) and (1.7). We begin with the proof of (1.6).

\subsection{Proof of (1.6)}

In order to avoid oscillations of $u$ when $|u| \simeq 1$ we first reproject $u$. To that aim let $f: \mathbb{R}^{+} \rightarrow[1,2]$ be a function such that $f(t)=\frac{1}{t}$ if $t \geq 1 / 2, f(t)=1$ if $t \leq 1 / 4$ and $\left|f^{\prime}\right| \leq 4$. Set

$$
\tilde{u}=f(|u|) \cdot u \quad \text { on } \quad B_{1} .
$$

Notice that

$$
J \tilde{u}=d\left(f^{2}(|u|) u \times d u\right) \quad \text { and } \quad\left|1-f^{2}(|u|)\right| \leq C\left(1-|u|^{2}\right) .
$$

Moreover, a property which is central in the proof is that

$$
|J \tilde{u}| \leq C \frac{\left(1-|u|^{2}\right)^{2}}{\varepsilon^{2}}=C V_{\varepsilon}\left(u_{\varepsilon}\right) .
$$

Indeed, if $|u| \geq 1 / 2$, then by construction $J \tilde{u}=0$. On the other hand, if $|u| \leq 1 / 2$, then $V_{\varepsilon}\left(u_{\varepsilon}\right) \geq \frac{1}{64 \varepsilon^{2}}$, whereas $|J \tilde{u}| \leq \frac{C}{\varepsilon^{2}}$, as a consequence of estimate (2.4).

Let us show next that the difference $J \tilde{u}-J u$ is small in a suitable norm.

Step 1: comparing $J \tilde{u}$ to $J u$. We claim that for $1 \leq p<2$,

$$
\|J \tilde{u}-J u\|_{W^{-1, p\left(B_{7 / 8}\right)}}^{p} \leq C_{p} \varepsilon^{2-p} E_{\varepsilon}\left(u_{\varepsilon}\right) .
$$

In particular, if $\tilde{\psi}_{1}$ is the solution of $\Delta \tilde{\psi}_{1}=\chi J \tilde{u}$ on $\mathbb{R}^{N}$, then we have,

$$
\int\left|\nabla\left(\tilde{\psi}_{1}-\psi_{1}\right)\right|^{p} \leq C_{p} \varepsilon^{2-p} E_{\varepsilon}\left(u_{\varepsilon}\right) .
$$


Proof. We have $J \tilde{u}-J u=d\left(\left(1-f^{2}(|u|)\right) u \times d u\right)$, and

$$
\begin{aligned}
\left\|\left(1-f^{2}(|u|)\right) u \times d u\right\|_{L^{p}\left(B_{7 / 8}\right)}^{p} & \leq C \int_{B_{7 / 8}}\left(1-|u|^{2}\right)^{p}|\nabla u|^{p} \\
& \leq C\left(\int_{B_{7 / 8}}\left(1-|u|^{2}\right)^{\frac{2 p}{2-p}}\right)^{1-\frac{p}{2}} E_{\varepsilon}\left(u_{\varepsilon}\right)^{p / 2} \\
& \leq C\left(\int_{B_{7 / 8}}\left(1-|u|^{2}\right)^{2}\right)^{1-\frac{p}{2}} E_{\varepsilon}\left(u_{\varepsilon}\right)^{p / 2} \\
& \leq C \varepsilon^{2-p} E_{\varepsilon}\left(u_{\varepsilon}\right),
\end{aligned}
$$

so that (4.12) and (4.13) follow.

In order to handle $\tilde{\psi}_{1}$, we consider the (vector) measure $\mu$ defined by

$$
\mu=\chi J \tilde{u} d x \quad \text { on } \quad \mathbb{R}^{N} .
$$

In view of (4.11) we have

$$
|\mu| \leq C \chi V_{\varepsilon}\left(u_{\varepsilon}\right) d x \quad \text { on } \quad \mathbb{R}^{N} .
$$

Next we consider, for $q>2$, the maximal fractional operator (see e.g. [38], p.204)

$$
M_{q}|\mu|(x)=\sup \left\{r^{q-N}|\mu|(B(x, r)), r>0\right\}
$$

Step 2: estimate of ||$M_{q}|\mu| \|_{L^{\infty}\left(\mathbb{R}^{N}\right)}$. We have, for every $q>2$,

$$
\left\|M_{q}|\mu|\right\|_{L^{\infty}} \leq C_{q} \Lambda\left(\frac{E_{\varepsilon}\left(u_{\varepsilon}\right)}{|\log \varepsilon|}\right) .
$$

Proof of (4.16). In view of the definition of $\mu$ we have, by Theorem 1.1

$$
|\mu|\left(\mathbb{R}^{N}\right) \leq \int_{B_{7 / 8}} V_{\varepsilon}\left(u_{\varepsilon}\right) \leq C \Lambda\left(\frac{E_{\varepsilon}\left(u_{\varepsilon}\right)}{|\log \varepsilon|}\right) .
$$

Next, for $x \in \mathbb{R}^{N}$ and $\varepsilon^{1 / 2} \leq r \leq 1 / 16$ we have, by (3.15),

$$
|\mu|(B(x, r)) \leq \frac{C}{|\log \varepsilon|} \log \left(2+\frac{E_{\varepsilon}\left(u_{\varepsilon}\right)}{|\log \varepsilon|}\right)(1+|\log r|) \int_{B(x, r)} e_{\varepsilon}\left(u_{\varepsilon}\right) .
$$

Combining (4.18) with the monotonicity formula, we are led to the inequality, for $x \in \mathbb{R}^{N}, \varepsilon^{1 / 2} \leq r \leq 1 / 16$,

$$
|\mu|(B(x, r)) \leq C r^{N-2}(1+|\log r|) \Lambda\left(\frac{E_{\varepsilon}\left(u_{\varepsilon}\right)}{|\log \varepsilon|}\right) .
$$


Finally, for small radii $0<r<\varepsilon^{1 / 2}$, we have the straightforward estimate

$$
|\mu|(B(x, r)) \leq \int_{B(x, r)} \chi e_{\varepsilon}\left(u_{\varepsilon}\right) \leq C r^{N-2} E_{\varepsilon}\left(u_{\varepsilon}\right),
$$

so that, in this case

$$
r^{q-N}|\mu|(B(x, r)) \leq C \varepsilon^{\frac{q-2}{2}} E_{\varepsilon}\left(u_{\varepsilon}\right) .
$$

Combining (4.17), (4.18) and (4.20), (4.16) follows.

Step 3: estimate for $\tilde{\psi}_{1}$. Recall that

$$
\Delta \tilde{\psi}_{1}=\mu \quad \text { on } \quad \mathbb{R}^{N} .
$$

By Theorem 4.7.4 of [38] ${ }^{(16)}$, for every $1<q_{0}<q_{1}<N$ we have

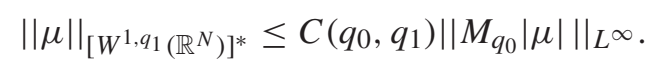

By duality, since $\mu$ is compactly supported in $B_{1}$ it follows

$$
\left\|\nabla \tilde{\psi}_{1}\right\|_{L^{q_{1}^{\prime}\left(B_{1}\right)}} \leq C\left(q_{0}, q_{1}\right)\left\|M_{q_{0}}|\mu|\right\|_{L^{\infty}} .
$$

Finally we choose $q_{1}^{\prime}=p$ so that $q_{1}>2$. Choosing $q_{0}=\frac{q_{1}+2}{2}$ we have $q_{0}>2$ so that we may estimate the right-hand side of (4.21) by Step 3. this yields

$$
\left\|\nabla \tilde{\psi}_{1}\right\|_{L^{p}\left(B_{1}\right)} \leq C_{p} \Lambda\left(\frac{E_{\varepsilon}\left(u_{\varepsilon}\right)}{|\log \varepsilon|}\right) .
$$

Step 4: proof of (1.6) completed. Combining (4.9), (4.13) and (4.22) we obtain

$$
\left\|\nabla \psi_{1}\right\|_{L^{p}\left(B_{6 / 7}\right)} \leq C_{p} \Lambda\left(\frac{E_{\varepsilon}\left(u_{\varepsilon}\right)}{|\log \varepsilon|}\right) .
$$

Combining (4.23) with (4.9), we deduce

$$
\|\nabla \psi\|_{L^{p}\left(B_{6 / 7}\right)} \leq C_{p}\left(\Lambda\left(\frac{E_{\varepsilon}\left(u_{\varepsilon}\right)}{|\log \varepsilon|}\right)+\|\nabla u\|_{L^{1}\left(B_{8 / 9}\right)}\right),
$$

and hence by (4.8)

$$
\|\nabla \Phi\|_{L^{\infty}(5 / 6)} \leq C\left(\Lambda\left(\frac{E_{\varepsilon}\left(u_{\varepsilon}\right)}{|\log \varepsilon|}\right)+\|\nabla u\|_{L^{1}\left(B_{8 / 9}\right)}\right) .
$$

(16) which goes back to results in [1]. See also related issues in [26, 2, 31]. 
Going back to (4.7) we are led to

$$
\| u \times\left.\nabla u\right|_{L^{p}\left(B_{6 / 7}\right)} \leq C_{p}\left(\Lambda\left(\frac{E_{\varepsilon}\left(u_{\varepsilon}\right)}{|\log \varepsilon|}\right)+\|\nabla u\|_{L^{1}\left(B_{8 / 9}\right)}\right) .
$$

Next we distinguish two cases.

Case 1. $E_{\varepsilon}\left(u_{\varepsilon}\right) \leq \eta_{1}|\log \varepsilon|$, where $\eta_{1}$ is the constant appearing in Theorem A.7 of the Appendix. Then we may apply Theorem A.7, and (1.6) is an immediate consequence of (A.39).

Case 2. $E_{\varepsilon}\left(u_{\varepsilon}\right) \geq \eta_{1}|\log \varepsilon|$. Then (1.6) follows combining (4.2), (4.3), (4.4) and (4.24).

\subsection{Proof of Theorem 1.5}

As mentioned in the introduction, and in view of Theorem A.7 it suffices to consider the case

$$
\eta_{1}|\log \varepsilon| \leq E_{\varepsilon}\left(u_{\varepsilon}\right) \leq|\log \varepsilon|^{2},
$$

where $\eta_{1}$ is the constant appearing in Theorem A.7. We therefore assume (4.25) holds throughout this section. The main difference with the proof of (1.6) is the treatment of equation (4.10), and the way we approximate the Jacobian $J u_{\varepsilon}$. The main new ingredient is an approximation procedure, taken from [13]. The monotonicity formula, which is crucial in the above proof of (1.6), (see e.g. (4.20)) does not hold however for the approximating map, at least at very small scales. In order to overcome this difficulty we smooth out $\Psi$ using a convolution by a mollifier. More precisely, we introduce a small scale $\delta_{0}$ of the form

$$
\delta_{0}=\delta_{0}(\varepsilon)=\left(\frac{1}{|\log \varepsilon|}\right)^{k_{0}}
$$

where $k_{0}>0$ will be determined later. Let $\zeta$ a smooth positive function compactly supported in $B_{1}$ such that $\int_{B_{1}} \zeta=1$, and set

$$
\zeta_{\varepsilon}(x)=\delta_{0}^{-N} \zeta\left(\frac{x}{\delta_{0}}\right)
$$

We introduce next the 2-form

$$
\tilde{\psi}_{1}=\psi_{1} * \zeta_{\varepsilon}
$$

so that $\tilde{\psi}_{1}$ satisfies the equation

$$
\Delta \tilde{\psi}_{1}=\left(\chi J u_{\varepsilon}\right) * \zeta_{\varepsilon} \quad \text { on } \quad \mathbb{R}^{N} .
$$

The first observation is that $\tilde{\psi}_{1}$ is close to $\psi_{1}$. 
4.3.1. Comparing $\nabla \tilde{\psi}_{1}$ to $\nabla \psi_{1}$

Proposition 4.1. We have

$$
\begin{gathered}
\left\|\nabla \tilde{\psi}_{1}-\nabla \psi_{1}\right\|_{L^{1}} \leq C \delta_{0} E_{\varepsilon}\left(u_{\varepsilon}\right), \\
\left\|\nabla \psi_{1}\right\|_{L^{2}}+\left\|\nabla \tilde{\psi}_{1}\right\|_{L^{2}} \leq C E_{\varepsilon}\left(u_{\varepsilon}\right)^{1 / 2}
\end{gathered}
$$

so that for any $1 \leq p<2$ we have by interpolation

$$
\left\|\nabla \tilde{\psi}_{1}-\nabla \psi_{1}\right\|_{L^{p}} \leq C_{p} \delta_{0}^{\frac{2}{p}-1} E_{\varepsilon}\left(u_{\varepsilon}\right)^{1 / p}
$$

Proof. Recall that for any function $w$ and $1 \leq p<+\infty$,

$$
\left\|w * \zeta_{\varepsilon}-w\right\|_{L^{p}} \leq C_{p} \delta_{0}\|\nabla w\|_{L^{p}}
$$

We apply (4.30) to $w=\nabla \psi_{1}, p=1$. To that aim, we claim

$$
\left\|\nabla \psi_{1}\right\|_{W^{1,1}} \leq C E_{\varepsilon}\left(u_{\varepsilon}\right)
$$

Proof of the claim. It follows from a result in [23] that if $v \in H^{1}\left(\mathbb{R}^{N} ; \mathbb{C}\right)$, then the Jacobian $J v$ belongs to the Hardy space $\mathcal{H}^{1}$, and that

$$
\|J v\|_{\mathcal{H}^{1}\left(\mathbb{R}^{N}\right)} \leq C\|\nabla v\|_{L^{2}\left(\mathbb{R}^{N}\right)} .
$$

On the other hand, a classical result in harmonic analysis states that if $f \in \mathcal{H}^{1}\left(\mathbb{R}^{N}\right)$, then

$$
\left\|\nabla^{2}\left(\Delta^{-1} f\right)\right\|_{L^{1}\left(\mathbb{R}^{N}\right)} \leq C\|f\|_{\mathcal{H}^{1}\left(\mathbb{R}^{N}\right)}
$$

We apply (4.32) to a suitable extension of $u_{\varepsilon}$ to the whole of $\mathbb{R}^{N}$, so that by (4.33) and a few auxiliary computations we deduce

$$
\left\|\nabla^{2}\left(\Delta^{-1}\left(\chi J u_{\varepsilon}\right)\right)\right\|_{L^{1}\left(\mathbb{R}^{N}\right)} \leq C\left\|\nabla u_{\varepsilon}\right\|_{L^{2}\left(\mathbb{R}^{N}\right)},
$$

and the claim follows.

Inequality (4.27) is then a direct consequence of (4.31), whereas for (4.28) we invoke the identity $J u_{\varepsilon}=d\left(u_{\varepsilon} \times d u_{\varepsilon}\right)$. 
4.3.2. Approximation with vorticity bounds

The main result of [13] is

Proposition 4.2. Let $0<\gamma<1$ and $u_{\varepsilon}: B_{1} \rightarrow \mathbb{C}$ be such that $E_{\varepsilon}\left(u_{\varepsilon}\right) \leq \varepsilon^{-\gamma}$. There exists constants $C>0$ and $\alpha>0$ depending only on $\gamma$ and $N$ such that there exists a smooth function $v_{\varepsilon}: B_{1} \rightarrow \mathbb{C}$ such that

$$
\begin{gathered}
\left|v_{\varepsilon}\right| \leq 1, \quad E_{\varepsilon}\left(v_{\varepsilon}\right) \leq C E_{\varepsilon}\left(u_{\varepsilon}\right) \\
\left\|J v_{\varepsilon}\right\|_{L^{1}} \leq C \frac{E_{\varepsilon}\left(u_{\varepsilon}\right)}{|\log \varepsilon|} \\
\left\|v_{\varepsilon}-u_{\varepsilon}\right\|_{L^{2}\left(B_{1}\right)} \leq C \varepsilon^{\alpha} E_{\varepsilon}\left(u_{\varepsilon}\right)^{1 / 2} .
\end{gathered}
$$

Notice in particular that if $u_{\varepsilon}$ and $v_{\varepsilon}$ are as above, then

$$
J u_{\varepsilon}=J v_{\varepsilon}+\kappa_{\varepsilon}
$$

where

$$
\kappa_{\varepsilon} \equiv d h_{\varepsilon} \equiv d\left(\left(u_{\varepsilon}-v_{\varepsilon}\right) \times d\left(u_{\varepsilon}+v_{\varepsilon}\right)\right) .
$$

If $u_{\varepsilon}$ itself is uniformly bounded, we have the following

Lemma 4.3. Assume $u_{\varepsilon}$ is as in Proposition 4.2 and verifies moreover

$$
\left|u_{\varepsilon}\right| \leq 2 \quad \text { on } \quad B_{1} \text {. }
$$

Then, for any $1 \leq p<2$, we have

$$
\left\|h_{\varepsilon}\right\|_{L^{p}} \leq C_{p} \varepsilon^{\alpha_{p}} E_{\varepsilon}\left(u_{\varepsilon}\right)^{1 / p}
$$

where $C_{p}>0$ and $0<\alpha_{p}<1$ depend only on $p$.

Comment. In the case considered above, $J u_{\varepsilon}$ has been split in a term bounded in $L^{1}$ by $\frac{E_{\varepsilon}\left(u_{\varepsilon}\right)}{|\log \varepsilon|}$ and a term which is small in $W^{-1, p}$, for every $1 \leq p<2$.

Proof of Lemma 4.3. Since by (4.34) and assumption (4.39) $\left\|u_{\varepsilon}-v_{\varepsilon}\right\|_{L^{\infty}} \leq 3$ it follows by interpolation and (4.36) that, for any $r \geq 2$,

$$
\left\|u_{\varepsilon}-v_{\varepsilon}\right\|_{L^{r}\left(B_{1}\right)} \leq\left\|u_{\varepsilon}-v_{\varepsilon}\right\|_{L^{2}\left(B_{1}\right)}^{2 / r}\left\|u_{\varepsilon}-v_{\varepsilon}\right\|_{L^{\infty}\left(B_{1}\right)}^{(r-2) / r} \leq C \varepsilon^{2 \alpha / r} E_{\varepsilon}\left(u_{\varepsilon}\right)^{1 / r} .
$$

Hence, for $1 \leq p<2$,

$$
\left\|h_{\varepsilon}\right\|_{L^{p}\left(B_{1}\right)} \leq\left\|u_{\varepsilon}-v_{\varepsilon}\right\|_{L^{2 p /(p-2)}\left(B_{1}\right)} E_{\varepsilon}\left(u_{\varepsilon}\right)^{p / 2} \leq C \varepsilon^{(2-p) \alpha} E_{\varepsilon}\left(u_{\varepsilon}\right),
$$

so that the conclusion follows.

We provide next a more localized version of Proposition 4.2. 
Proposition 4.4. Let $1 \leq p<2$ and assume $u_{\varepsilon}$ satisfies (4.25). Let $k_{1}>0$ and set $\delta_{1}=\delta_{1}(\varepsilon)=|\log \varepsilon|^{-k_{1}}$. Then we may decompose $\chi J u_{\varepsilon}$ as

$$
\chi J u_{\varepsilon}=v_{\varepsilon}+r_{\varepsilon}
$$

where $v_{\varepsilon}$ is compactly supported in $B_{7 / 8}$ and satisfies, for some constant $C\left(k_{1}\right)>0$ depending only on $N$ and $k_{1}$,

$$
\sup \left\{r^{2-N}\left|v_{\varepsilon}\right| B(x, r), x \in \mathbb{R}^{N}, r \geq \delta_{1}(\varepsilon)\right\} \leq C\left(k_{1}\right) \frac{E_{\varepsilon}\left(u_{\varepsilon}\right)}{|\log \varepsilon|},
$$

and where $r_{\varepsilon}$ satisfies, for some $0<\beta<1$ depending only on $p$ and $k_{1}$,

$$
\left\|r_{\varepsilon}\right\|_{W^{-1, p}\left(B_{1}\right)} \leq C_{p} \varepsilon^{\beta} E_{\varepsilon}\left(u_{\varepsilon}\right)^{1 / p} .
$$

Proof. The argument is to apply Proposition 4.2 on balls of size $\delta_{1}(\varepsilon)$ and then reconnect the resulting functions thanks to a partition of unity. More precisely, cover $B_{1}$ by balls $B_{i} \equiv B\left(x_{i}, \delta_{1}(\varepsilon)\right), i=1, \ldots, \ell_{\varepsilon}$ such that the balls $B\left(x_{i}, \delta_{1} / 8\right)$ do not intersect, and consider a partition of unity $\left\{\chi_{i}\right\}_{1 \leq i \leq \ell_{\varepsilon}}$ for the previous covering, i.e. such that $\chi_{i}$ is smooth, compactly supported in $B\left(x_{i}, \delta_{1}\right)$ and moreover

$$
\left|\nabla \chi_{i}\right| \leq \frac{C}{\delta_{1}}
$$

for some constant $C$ depending only on $N$.

On each ball $B_{i}$ we apply Proposition 4.2 to the restriction of $u_{\varepsilon}$, after a suitable change of scales. This yields maps $v_{\varepsilon}^{i}: B_{i} \rightarrow \mathbb{C}$ such that $\left|v_{\varepsilon}^{i}\right| \leq 1$ and

$$
\int_{B_{i}} e_{\varepsilon}\left(v_{\varepsilon}^{i}\right) \leq C \int_{B_{i}} e_{\varepsilon}\left(u_{\varepsilon}\right), \quad \int_{B_{i}}\left|J v_{\varepsilon}^{i}\right| \leq \frac{C}{|\log \varepsilon|} \int_{B_{i}} e_{\varepsilon}\left(u_{\varepsilon}\right)
$$

and

$$
\left\|u_{\varepsilon}-v_{\varepsilon}\right\|_{L^{2}\left(B_{i}\right)}^{2} \leq C \delta_{1}^{2} \varepsilon^{2 \alpha} \int_{B_{i}} e_{\varepsilon}\left(u_{\varepsilon}\right)
$$

Moreover, for $\kappa_{\varepsilon}^{i}=J u_{\varepsilon}-J v_{\varepsilon}^{i}=d h_{i}=d\left(\left(u_{\varepsilon}-v_{\varepsilon}\right) \times d\left(u_{\varepsilon}+v_{\varepsilon}\right)\right)$ we have, for every $1 \leq p<2$,

$$
\left\|h_{i}\right\|_{L^{p}\left(B_{i}\right)} \leq C_{p} \delta_{1}^{(2-p) / p} \varepsilon^{\alpha_{p}}\left(\int_{B_{i}} e_{\varepsilon}\left(u_{\varepsilon}\right)\right)^{1 / p} .
$$

Setting $v_{\varepsilon}=\chi\left(\sum_{i} \chi_{i} J v_{\varepsilon}^{i}\right)$, we may write $\chi J u_{\varepsilon}=v_{\varepsilon}+r_{\varepsilon}$, with

$$
r_{\varepsilon}=\chi\left(\sum_{i=1}^{\ell_{\varepsilon}} \chi_{i} \kappa_{\varepsilon}^{i}\right)=\sum_{i=1}^{\ell_{\varepsilon}} d\left(\chi \chi_{i} h_{\varepsilon}^{i}\right)+\sum_{i=1}^{\ell_{\varepsilon}} d\left(\chi \chi_{i}\right) h_{\varepsilon}^{i}
$$


In view of (4.46) and (4.43), we derive (4.42). Concerning $v_{\varepsilon}$, we have to bound uniformly $r^{2-N} \int_{B(x, r)}\left|v_{\varepsilon}\right|$. Firstly we notice that by (4.44) and the properties of the covering, we have, for sufficiently small $\varepsilon$ and $r \geq \delta_{1}(\varepsilon)$

$$
\int_{B(x, r)}\left|v_{\varepsilon}\right| \leq \frac{C}{|\log \varepsilon|} \int_{B\left(x_{i}, 8 r\right)} \tilde{\chi} e_{\varepsilon}\left(u_{\varepsilon}\right),
$$

where $\tilde{\chi} \geq \chi$ is some smooth function with compact support in $B_{8 / 9}$. Hence, by monotonicity,

$$
\sup \left\{r^{2-N} \int_{B(x, r)}\left|v_{\varepsilon}\right|, r \geq \delta_{1}, x \in \mathbb{R}^{N}\right\} \leq C \frac{E_{\varepsilon}\left(u_{\varepsilon}\right)}{|\log \varepsilon|}
$$

and the proof is complete.

We now turn to the measure $\tilde{v}_{\varepsilon}=v_{\varepsilon} * \zeta_{\varepsilon}$.

Lemma 4.5. Assume $k_{0}=k_{1}$, i.e. $\delta_{0}(\varepsilon)=\delta_{1}(\varepsilon)$. Then we have

$$
\left\|M_{2}\left|\tilde{v}_{\varepsilon}\right|\right\|_{L^{\infty}\left(\mathbb{R}^{N}\right)} \leq C \frac{E_{\varepsilon}\left(u_{\varepsilon}\right)}{|\log \varepsilon|}
$$

where the constant $C$ depends only on $k_{0}=k_{1}$.

Proof. In view of the definition of $\tilde{v}_{\varepsilon}$ we have, for any $y \in \mathbb{R}^{N}$,

$$
\left|\tilde{v}_{\varepsilon}(y)\right| \leq C \delta_{0}^{-N} \int_{B\left(x, \delta_{0}\right)}\left|v_{\varepsilon}\right|
$$

In order to bound $r^{2-N} \int_{B(x, r)}\left|\tilde{v}_{\varepsilon}(y)\right| d y$ uniformly on $\mathbb{R}$, we distinguish two cases.

Case 1: $r \leq \delta_{0}$. By Proposition 4.2 and (4.49), we have

$$
\left|\tilde{\nu}_{\varepsilon}(y)\right| \leq C \delta_{0}^{-2} \frac{E_{\varepsilon}\left(u_{\varepsilon}\right)}{|\log \varepsilon|} .
$$

Integrating, we deduce

$$
r^{2-N} \int_{B(x, r)}\left|\tilde{v}_{\varepsilon}(y)\right| \leq C r^{2} \delta_{0}^{-2} \frac{E_{\varepsilon}\left(u_{\varepsilon}\right)}{|\log \varepsilon|} \leq C \frac{E_{\varepsilon}\left(u_{\varepsilon}\right)}{|\log \varepsilon|} .
$$


Case 2: $r \geq \delta_{0}$. We write

$$
\begin{aligned}
\int_{B(x, r)}\left|\tilde{v}_{\varepsilon}(y)\right| d y & =\int_{B(x, r)}\left|\int_{B\left(0, \delta_{0}\right)} \zeta_{\varepsilon}(z) v_{\varepsilon}(y-z) d z\right| d y \\
& \leq \int_{B(x, r)} \int_{B\left(0, \delta_{0}\right)} \zeta_{\varepsilon}(z)\left|v_{\varepsilon}\right|(y-z) d z d y \\
& =\int_{B\left(0, \delta_{0}\right)} \int_{B(x, r)} \zeta_{\varepsilon}(z)\left|v_{\varepsilon}\right|(y-z) d y d z \\
& =\int_{B\left(0, \delta_{0}\right)} \zeta_{\varepsilon}(z)\left(\int_{B(x-z, r)}\left|v_{\varepsilon}(w)\right| d w\right) d z \\
& \leq C r^{N-2} \frac{E_{\varepsilon}\left(u_{\varepsilon}\right)}{|\log \varepsilon|}
\end{aligned}
$$

where we invoke Proposition 4.4 for the last inequality. Combining the two cases, the conclusion follows.

\subsubsection{Proof of Theorem 1.5 completed}

The main difference with the proof of (1.6) is that instead of (4.23) we are now in position to use the stronger estimate, if $u_{\varepsilon}$ verifies (4.25),

$$
\left\|\nabla \psi_{1}\right\|_{L^{p}\left(B_{6 / 7}\right)} \leq C_{p} \frac{E_{\varepsilon}\left(u_{\varepsilon}\right)}{|\log \varepsilon|} .
$$

When (4.51) is established, then the proof carries out almost verbatim.

Proof of (4.51). We determine the constant $k_{0}$ in (4.26), going back to (4.29). Indeed, choosing

$$
k_{0}=k_{1}=\left(1-\frac{2}{p}\right)^{2},
$$

we obtain by (4.29)

$$
\left\|\nabla \tilde{\psi}_{1}-\nabla \psi_{1}\right\|_{L^{p}} \leq C_{p} \frac{E_{\varepsilon}\left(u_{\varepsilon}\right)}{|\log \varepsilon|}
$$

Next, we write $\tilde{\psi}_{1}=\tilde{\psi}_{1}^{0}+\tilde{\psi}_{1}^{1}$, where $\tilde{\psi}_{1}^{0}$ is the solution obtained by convolution with the fundamental solution of the Laplacian of

$$
\Delta \tilde{\psi}_{1}^{0}=\tilde{v}_{\varepsilon} \quad \text { on } \quad \mathbb{R}^{N},
$$

and where similarly $\tilde{\psi}_{1}^{1}$ solves

$$
\Delta \tilde{\psi}_{1}^{0}=\tilde{r}_{\varepsilon} \equiv r_{\varepsilon} * \zeta_{\varepsilon} \quad \text { on } \quad \mathbb{R}^{N} .
$$


In view of Lemma 4.5 and invoking once more Theorem 4.7.4 of [38], we obtain

$$
\left\|\nabla \tilde{\psi}_{1}^{0}\right\|_{L^{p}} \leq\left\|M_{2}\left|\tilde{v}_{\varepsilon}\right|\right\|_{L^{\infty}} \leq C_{p} \frac{E_{\varepsilon}\left(u_{\varepsilon}\right)}{|\log \varepsilon|} .
$$

By (4.42) in Proposition 4.2, we deduce

$$
\left\|\nabla \tilde{\psi}_{1}^{1}\right\|_{L^{p}} \leq C_{p} \varepsilon^{\beta} E_{\varepsilon}\left(u_{\varepsilon}\right) \leq C_{p} \frac{E_{\varepsilon}\left(u_{\varepsilon}\right)}{|\log \varepsilon|},
$$

so that combining (4.52), (4.53) and (4.54), we obtain (4.51).

\section{Appendix}

In this Appendix we consider the following situation. Consider the unit ball $B_{1} \subset$ $\mathbb{R}^{N}$, and $u_{\varepsilon}$ a solution to $(\mathrm{GL})_{\varepsilon}$ verifying

$$
\left|u_{\varepsilon}\right| \geq \frac{1}{2} \quad \text { on } \quad B_{1},
$$

or the even more restrictive assumption

$$
\left|u_{\varepsilon}\right| \geq 1-\sigma_{0}
$$

where $0<\sigma_{0}<1 / 2$ is some constant which will be introduced later. In particular we may write

$$
u_{\varepsilon}=\rho_{\varepsilon} e^{i \varphi_{\varepsilon}} \quad \text { on } \quad B_{1}
$$

where $\varphi_{\varepsilon}$ is a real-valued function defined on $B_{1}$ and $\rho_{\varepsilon}$ denotes the modulus $\left|u_{\varepsilon}\right|$ of $u_{\varepsilon}$. Our aim is to derive a number of improved estimates, in particular pointwise estimates, under the additional assumption (A.1). This will lead us among other things to the proof of Theorem 2.1.

\section{A.1. Pointwise estimates}

We first have

Proposition A.1. Let $u_{\varepsilon}$ be a solution of $(G L)_{\varepsilon}$ on $B_{1}$ verifying (A.1), then

$$
\left|u_{\varepsilon}\right| \geq 1-C \varepsilon^{2}\left(1+\left\|\nabla \varphi_{\varepsilon}\right\|_{\left.L_{\left(B_{\frac{3}{4}}\right)}^{2}\right)}^{\text {on }} \quad B_{\frac{1}{2}}\right.
$$

where $C>0$ is some constant depending only on $N$. 
Proof. The equation for $\theta_{\varepsilon}=1-\rho_{\varepsilon}$ writes

$$
-\Delta \theta_{\varepsilon}+a \theta_{\varepsilon}=b
$$

where we have set

$$
a=\frac{1+\left(1-\theta_{\varepsilon}\right)^{2}}{\varepsilon^{2}}, \quad b=\left(1-\theta_{\varepsilon}\right)\left|\nabla \varphi_{\varepsilon}\right|^{2} .
$$

We can conclude applying the maximum principle as in the Appendix of [14].

Our next purpose is to obtain pointwise bounds for $\left|\nabla u_{\varepsilon}\right|$, and hence $\left|\nabla \varphi_{\varepsilon}\right|$. To that aim, we introduce a bound on the energy of the form

$$
E_{\varepsilon}\left(u_{\varepsilon}\right) \leq \varepsilon^{-\alpha},
$$

for $0<\alpha<1$. We then have

Theorem A.2. Let $u_{\varepsilon}$ be a solution of $(G L)_{\varepsilon}$ on $B_{1}$. There exist constants $0<$ $\alpha_{0}<1$ and $0<\sigma_{0} \leq \frac{1}{2}$ depending only on $N$ such that if $\left(H_{\alpha_{0}}\right)$ is satisfied and if

$$
\left|u_{\varepsilon}\right| \geq 1-\sigma_{0} \quad \text { on } \quad B_{1},
$$

then

$$
e_{\varepsilon}\left(u_{\varepsilon}\right)(x) \leq C \int_{B_{1}} e_{\varepsilon}\left(u_{\varepsilon}\right), \quad \text { on } \quad B_{\frac{3}{4}}
$$

where the constant $C$ depends only on $N$.

Remark A.3. In a more general context, Chen and Struwe [21] (see also [34]) established (A.7) replacing $\left(H_{\alpha}\right)$ by the stronger assumption

$$
E_{\varepsilon}\left(u_{\varepsilon}\right) \leq \gamma_{0},
$$

where $\gamma_{0}>0$ is some constant depending only on $N$. Our proof of Theorem A.2 will rely on the Chen-Struwe result in an essential way. The main point is to show that (A.8) is met locally, after suitable scalings.

Proof of Theorem A.2. Changing $u_{\varepsilon}$ possibly by a constant phase, we may impose the additional condition

$$
\frac{1}{\left|B_{1}\right|} \int_{B_{1}} \varphi_{\varepsilon}=0
$$

Inserting (A.2) into (GL) $)_{\varepsilon}$ we are led to the elliptic equation

$$
-\operatorname{div}\left(\rho_{\varepsilon}^{2} \nabla \varphi_{\varepsilon}\right)=0 \quad \text { in } B_{1} .
$$


In contrast with the equation for the modulus, (A.10) has the advantage that the explicit dependence on $\varepsilon$ has been removed. We will handle (A.10) as a linear equation for the function $\varphi_{\varepsilon}, \rho_{\varepsilon}$ being considered as a coefficient. In the sequel, we write $\varphi=\varphi_{\varepsilon}$ and $\rho=\rho_{\varepsilon}$ when this is not misleading. In order to avoid boundary conditions, we consider the truncated function $\tilde{\varphi}$ defined by $\tilde{\varphi}=\varphi \chi$, where $\chi$ is a smooth cut-off function such that

$$
\chi \equiv 1 \text { on } B_{\frac{4}{5}} \quad \text { and } \quad \chi \equiv 0 \text { on } \mathbb{R}^{N} \backslash B_{\frac{5}{6}} .
$$

The function $\tilde{\varphi}$ then verifies the equation

$$
-\operatorname{div}\left(\rho^{2} \nabla \tilde{\varphi}\right)=\operatorname{div}\left(\rho^{2} \varphi \nabla \chi\right)+\rho^{2} \nabla \chi \cdot \nabla \varphi \quad \text { in } B_{1} .
$$

Moreover, by construction

$$
\operatorname{supp}(\tilde{\varphi}) \subset B_{\frac{5}{6}} \cdot
$$

Since by assumption $\rho$ is close to 1 , it is natural to treat the 1.h.s. of (A.11) as a perturbation of the Laplace operator, and to rewrite (A.11) as follows

$$
-\Delta \tilde{\varphi}=\operatorname{div}\left(\left(\rho^{2}-1\right) \nabla \tilde{\varphi}\right)+\operatorname{div}\left(\rho^{2} \varphi \nabla \chi\right)+\rho^{2} \nabla \chi \cdot \nabla \varphi \quad \text { in } B_{\frac{5}{6}} .
$$

We introduce the function $\varphi_{0}$ defined on $B_{\frac{5}{6}}$ as the solution of

$$
\left\{\begin{aligned}
-\Delta \varphi_{0} & =\operatorname{div}\left(\rho^{2} \varphi \nabla \chi\right)+\rho^{2} \nabla \chi \cdot \nabla \varphi & & \text { in } B_{\frac{5}{6}} \\
\varphi_{0} & =0 & & \text { on }{ }^{2} B_{\frac{5}{6}}
\end{aligned}\right.
$$

In particular, since $\chi \equiv 1$ on $B\left(\frac{4}{5}\right)$, we have

$$
-\Delta \varphi_{0}=0 \quad \text { in } B_{4 / 5}
$$

We set $\varphi_{1}=\tilde{\varphi}-\varphi_{0}$, i.e.

$$
\tilde{\varphi}=\varphi_{0}+\varphi_{1} .
$$

We will show that $\varphi_{1}$ is essentially a perturbation term. At this stage, we divide the estimates into several steps. We start with linear estimates for $\varphi_{0}$.

Step 1 : Estimates for $\varphi_{0}$. We claim that

$$
\left\|\nabla \varphi_{0}\right\|_{L^{2^{*}\left(B_{5}\right)}}^{2} \leq C_{1}\left[\int_{B_{1}} e_{\varepsilon}\left(u_{\varepsilon}\right)\right]
$$

and

$$
\left\|\nabla \varphi_{0}\right\|_{L^{\infty}\left(B_{\frac{3}{4}}\right)}^{2} \leq C_{2}\left[\int_{B_{1}} e_{\varepsilon}\left(u_{\varepsilon}\right)\right]
$$

where $2^{*}=\frac{2 N}{N-2}$ is the Sobolev exponent in dimension $N$. 
Proof. The first estimate follows from the linear theory for the Laplace operator, whereas the second follows from the first one and the fact that $\varphi_{0}$ is harmonic on $B(4 / 5)$.

Step 2: The equation for $\varphi_{1}$. The function $\varphi_{1}$ verifies the elliptic problem

$$
\left\{\begin{aligned}
-\Delta \varphi_{1} & =\operatorname{div}\left(\left(\rho^{2}-1\right) \nabla \tilde{\varphi}\right) & & \text { in } B_{\frac{5}{6}}, \\
\varphi_{1} & =0 & & \text { on } \partial B_{\frac{5}{6}} .
\end{aligned}\right.
$$

It is convenient to rewrite equation (A.16) as

$$
-\Delta \varphi_{1}=\operatorname{div}\left(\left(\rho^{2}-1\right) \nabla \varphi_{1}\right)+\operatorname{div}\left(g_{0}\right),
$$

where we have set $g_{0}=\left(\rho^{2}-1\right) \nabla \varphi_{0}$. Using (A.14) and by assumption $\left(\mathrm{H}_{\alpha_{0}}\right)$ we obtain, for any $2 \leq q<2^{*}$, the estimate for $g_{0}$

$$
\left\|g_{0}\right\|_{L^{q}\left(B_{\frac{5}{6}}\right)}^{q} \leq C \varepsilon^{\left(2-\alpha_{0}\right) \frac{\left(2^{*}-q\right)}{2^{*}}}\left\|e_{\varepsilon}\left(u_{\varepsilon}\right)\right\|_{L^{1}}^{\frac{q}{2}} .
$$

Indeed, since $\frac{2^{*} q}{2^{*}-q} \geq 2$ and $|\rho| \leq C$ on $B_{\frac{5}{6}}$,

$$
\begin{aligned}
\int_{B_{\frac{5}{6}}}\left|\rho^{2}-1\right|^{q}\left|\nabla \varphi_{0}\right|^{q} & \leq\left(\int_{B_{1}}\left|\nabla \varphi_{0}\right|^{2^{*}}\right)^{\frac{q}{2^{*}}}\left(\int_{B_{1}}\left|\rho^{2}-1\right|^{\frac{2^{*} q}{2^{*}-q}}\right)^{\frac{2^{*}-q}{2^{*}}} \\
& \leq C\left\|e_{\varepsilon}\left(u_{\varepsilon}\right)\right\|_{L^{1}}^{\frac{q}{2}} \varepsilon^{\frac{2\left(2^{*}-q\right)}{2^{*}}}\left(\int_{B_{1}} \frac{\left|\rho^{2}-1\right|^{2}}{4 \varepsilon^{2}}\right)^{\frac{2^{*}-q}{2^{*}}},
\end{aligned}
$$

and the conclusion (A.18) follows.

We now estimate $\varphi_{1}$ from (A.17) through a fixed point argument.

Step 3: The fixed point argument. Equation (A.17) may be rewritten as

$$
\varphi_{1}=\mathcal{T}\left(\operatorname{div}\left(\left(\rho^{2}-1\right) \nabla \varphi_{1}\right)\right)+\mathcal{T}\left(\operatorname{div} g_{0}\right),
$$

which is of the form

$$
(\mathrm{Id}-A) \varphi_{1}=b
$$

where $\mathcal{T}=\Delta^{-1}, \quad A$ is the linear operator $v \mapsto \mathcal{T}\left(\operatorname{div}\left(\left(\rho^{2}-1\right) \nabla v\right)\right)$ and $b=$ $\mathcal{T}\left(\operatorname{div} g_{0}\right)$. Consider the Banach space $X_{q}=W_{0}^{1, q}\left(B_{\frac{5}{6}}\right)$. It follows from the linear theory for $\mathcal{T}$ that $A: X_{q} \rightarrow X_{q}$ is linear continuous and that

$$
\|A\|_{\mathcal{L}\left(X_{q}\right)} \leq C(q)\|1-\rho\|_{L_{\left(B_{\frac{5}{6}}\right)}} .
$$

In particular, we may choose the constant $\sigma>0$ in (A.6) such that

$$
C(q)\|1-\rho\|_{L^{\infty}(B(1))} \leq C(q) \sigma_{0}<\frac{1}{2} .
$$


With this choice of $\sigma$, we deduce that $I-A$ is invertible on $X_{q}$ and

$$
\left\|\varphi_{1}\right\|_{X_{q}} \leq C\|b\|_{X_{q}} .
$$

Finally, by (A.18) we obtain

$$
\|b\|_{X_{q}}=\left\|\mathcal{T}\left(\operatorname{div} g_{0}\right)\right\|_{X_{q}} \leq C\left\|g_{0}\right\|_{L^{q}} \leq C \varepsilon^{\frac{\left(2-\alpha_{0}\right)\left(2^{*}-q\right)}{q 2^{2}}}\left\|e_{\varepsilon}\left(u_{\varepsilon}\right)\right\|_{L^{1}}^{\frac{1}{2}} .
$$

Going to (A.19) we deduce

$$
\left\|\nabla \varphi_{1}\right\|_{L^{q}\left(B_{5 / 6}\right)} \leq C \varepsilon^{\frac{\left(2-\alpha_{0}\right)\left(2^{*}-q\right)}{2^{*} q}}\left\|e_{\varepsilon}\left(u_{\varepsilon}\right)\right\|_{L^{1}\left(B_{1}\right)}^{\frac{1}{2}} .
$$

We now combine the estimates for $\varphi_{0}$ and $\varphi_{1}$.

Step 4: Improved integrability of $\nabla \tilde{\varphi}$. Since $\alpha_{0}<2$, we obtain, combining (A.14) and (A.20),

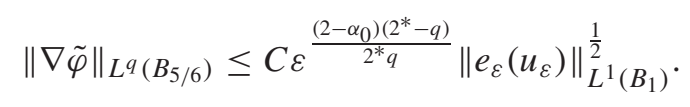

Comment. Since $q>2$, the previous estimate presents a substantial improvement over the corresponding inequality with $q$ replaced by 2 , which is immediate. This improvement is crucial in order to prove the smallness of both the modulus and potential terms in the energy, which we derive now.

Step 5: Estimates for the modulus and potential terms. Recall that the function $\rho$ satisfies the equation

$$
-\Delta \rho+\rho|\nabla \varphi|^{2}=\rho \frac{\left(1-\rho^{2}\right)}{\varepsilon^{2}} .
$$

Since $\chi \equiv 1$ on $B_{4 / 5}$, we have $\varphi=\tilde{\varphi}$ on $B_{4 / 5}$. Let $\xi$ be a non-negative cut-off function such that $\xi \equiv 1$ on $B_{3 / 4}$ and $\xi \equiv 0$ outside $B_{4 / 5}$. Multiplying (A.22) by $\left(1-\rho^{2}\right) \xi$ and integrating by parts we obtain

$$
\int_{B_{1}} 2 \rho|\nabla \rho|^{2} \xi+\int_{B_{1}} \rho \frac{\left(1-\rho^{2}\right)^{2}}{\varepsilon^{2}}=\int_{B_{1}} \nabla \rho \cdot \nabla \xi\left(1-\rho^{2}\right)+\int_{B_{1}} \rho\left(1-\rho^{2}\right)|\nabla \tilde{\varphi}|^{2} \xi .
$$

Hence, since $\rho \geq \frac{1}{2}$ on $B_{1}$ we obtain

$$
\begin{aligned}
\int_{B_{3 / 4}}|\nabla \rho|^{2}+V_{\varepsilon}\left(u_{\varepsilon}\right) \leq C \varepsilon\left(\int_{B_{1}}|\nabla \rho|^{2}\right)^{\frac{1}{2}}\left(\int_{B_{1}} V_{\varepsilon}\left(u_{\varepsilon}\right)\right)^{\frac{1}{2}} \\
+C\left(\int_{B_{4 / 5}}|\nabla \tilde{\varphi}|^{q}\right)^{\frac{2}{q}}\left(\int_{B_{4 / 5}}\left(1-\rho^{2}\right)^{\frac{q}{q-2}}\right)^{\frac{q-2}{q}}
\end{aligned}
$$


so that using (A.21) we finally infer that

$$
\int_{B_{3 / 4}}\left[|\nabla| u_{\varepsilon}||^{2}+V_{\varepsilon}\left(u_{\varepsilon}\right)\right] \leq C \varepsilon^{\beta_{q}} \int_{B_{1}} e_{\varepsilon}\left(u_{\varepsilon}\right)
$$

where $\beta_{q}=\left(4-2 \alpha_{0}\right) \frac{\left(2^{*}-q\right)}{2^{*} q}$. To summarize, we have proved at this stage that

$$
e_{\varepsilon}\left(u_{\varepsilon}\right) \leq\left|\nabla \varphi_{0}\right|^{2}+r_{\varepsilon}
$$

for some $r_{\varepsilon} \geq 0$ which verifies

$$
\int_{B_{\frac{3}{4}}} r_{\varepsilon} \leq C \varepsilon^{\beta_{q}} \int_{B_{1}} e_{\varepsilon}\left(u_{\varepsilon}\right)
$$

Step 6: Proof of Theorem A.2 completed. We are going to apply the result by Chen and Struwe [21] (see Remark A.3) to a suitably scaled version of $u_{\varepsilon}$.

Let $0<r_{0}<\frac{1}{8}$, to be determined later, set $\varepsilon=\frac{\varepsilon}{r_{0}}$ and let $x_{0} \in B_{\frac{1}{2}}$ be fixed. Consider the map $v_{\varepsilon}$ defined on $B_{1}$ by

$$
v_{\varepsilon}(x)=u_{\varepsilon}\left(\frac{x-x_{0}}{r_{0}}\right)
$$

so that $u_{\varepsilon}\left(x_{0}\right)=v_{\varepsilon}(0)$. By scaling, we have

$$
\int_{B_{1}} e_{\varepsilon}\left(v_{\varepsilon}\right)=\frac{1}{r_{0}^{N-2}} \int_{B\left(x_{0}, r_{0}\right)} e_{\varepsilon}\left(u_{\varepsilon}\right)
$$

Note in particular, since $r_{0}<\frac{1}{8}$, that $B\left(x_{0}, r_{0}\right) \subset B(3 / 4)$, and we may invoke the decomposition (A.24) to assert that

$$
\begin{aligned}
\int_{B\left(x_{0}, r_{0}\right)} e_{\varepsilon}\left(u_{\varepsilon}\right) & \leq \operatorname{meas}\left(B\left(x_{0}, r_{0}\right)\right) \cdot\left\|\nabla \varphi_{0}\right\|_{L^{\infty}}^{2}+\int_{B_{\frac{3}{4}}} r_{\varepsilon} \\
& \leq C r_{0}^{N}\left\|e_{\varepsilon}\left(u_{\varepsilon}\right)\right\|_{L^{1}\left(B_{1}\right)}+C \varepsilon^{\beta_{q}}\left\|e_{\varepsilon}\left(u_{\varepsilon}\right)\right\|_{L^{1}\left(B_{1}\right)}
\end{aligned}
$$

Hence, going back to (A.26)

$$
\int_{B_{1}} e_{\varepsilon}\left(v_{\varepsilon}\right) \leq C r_{0}^{2}\left\|e_{\varepsilon}\left(u_{\varepsilon}\right)\right\|_{L^{1}\left(B_{1}\right)}+C r_{0}^{2-N} \varepsilon^{\beta_{q}}\left\|e_{\varepsilon}\left(u_{\varepsilon}\right)\right\|_{L^{1}\left(B_{1}\right)},
$$

so that, in view of assumption $\left(H_{\alpha_{0}}\right)$,

$$
\int_{B_{1}} e_{\varepsilon}\left(v_{\varepsilon}\right) \leq C r_{0}^{2} \varepsilon^{-\alpha_{0}}+C r_{0}^{2-N} \varepsilon^{\beta_{q}-\alpha_{0}}
$$


We next determine the constants $q, \alpha_{0}$ and $r_{0}$ so that the right-hand side of (A.28) is less than $\gamma_{0}$. First, we choose $q$ arbitrarily in the interval $\left(2,2^{*}\right)$, say for instance $q=\frac{2+2^{*}}{2}$. We then notice that, varying $r_{0}$, the maximum of $r_{0}^{2} \varepsilon^{-\alpha_{0}}+r_{0}^{2-N} \varepsilon^{\beta_{q}-\alpha_{0}}$ is achieved for

$$
r_{0}=\left(\frac{N-2}{2}\right)^{1 / N} \varepsilon^{\beta_{q} / N}
$$

so that the right-hand side of (A.28) is less than $C \varepsilon^{2 \frac{\beta q}{N}-\alpha_{0}}$. Since $\beta_{q}=(4-$ $\left.2 \alpha_{0}\right) \frac{\left(2^{*}-q\right)}{2^{*} q}$, we may choose $\alpha_{0}$ sufficiently small so that $\beta_{q}>0$ and $2 \frac{\beta_{q}}{N}-\alpha_{0}>0$. In particular, for such a choice we have

$$
\int_{B_{1}} e_{\varepsilon}\left(v_{\varepsilon}\right) \leq \gamma_{0}
$$

for $\varepsilon$ sufficiently small. We are now in position to apply the Chern-Struwe result described in Remark A.3. This yields, in view of (A.27),

$$
\begin{aligned}
r_{0}^{2} e_{\varepsilon}\left(u_{\varepsilon}\right)\left(x_{0}\right) & =e_{\varepsilon}\left(v_{\varepsilon}\right)\left(x_{0}\right) \leq C \int_{B_{1}} e_{\varepsilon}\left(v_{\varepsilon}\right) \\
& \leq C r_{0}^{2}\left\|e_{\varepsilon}\left(u_{\varepsilon}\right)\right\|_{L^{1}\left(B_{1}\right)}+C r_{0}^{2-N} \varepsilon^{\beta_{q}}\left\|e_{\varepsilon}\left(u_{\varepsilon}\right)\right\|_{L^{1}\left(B_{1}\right)}
\end{aligned}
$$

and hence

$$
e_{\varepsilon}\left(u_{\varepsilon}\right)\left(x_{0}\right) \leq C \int_{B_{1}} e_{\varepsilon}\left(u_{\varepsilon}\right)
$$

and the proof is complete.

Notice that in the course of the proof, we have shown that the contribution of the modulus to the energy is small: More precisely, inequality (A.23) yields

Proposition A.4. Let $u_{\varepsilon}$ be a solution of $(G L)_{\varepsilon}$ on $B_{1}$. There exist constants $0<$ $\alpha_{0}<1$ and $0<\sigma_{0} \leq \frac{1}{2}$ depending only on $N$ such that if $\left(H_{\alpha_{0}}\right)$ is satisfied and if

$$
\left|u_{\varepsilon}\right| \geq 1-\sigma_{0} \quad \text { on } \quad B_{1},
$$

then

$$
\int_{B_{\frac{3}{4}}}\left[|\nabla| u_{\varepsilon}||^{2}+V_{\varepsilon}\left(u_{\varepsilon}\right)\right] \leq C \varepsilon^{\beta} \int_{B_{1}} e_{\varepsilon}\left(u_{\varepsilon}\right),
$$

where $\beta>0$ and $C$ depend only on $N$.

Remark A.5. Notice that we also have derived under assumption (A.32) a pointwise estimate for the potential in Proposition 2.3. In Theorem A.6 we also derive a pointwise bound for $|\nabla \rho|$. 
Proof of Theorem 2.1. Since we have the pointwise inequalities

$$
\left|\nabla \varphi_{\varepsilon}\right|^{2} \leq 4\left|\nabla u_{\varepsilon}\right|^{2} \leq 8 e_{\varepsilon}\left(u_{\varepsilon}\right)
$$

the conclusion of Theorem 2.1 in case $R=1$ follows combining (A.7) and (A.3). The general case is deduced by scaling.

Finally we derive pointwise estimates for derivatives. More precisely, we have

Theorem A.6. Assume that (A.2) and $\left(H_{\alpha_{0}}\right)$ hold, where $\alpha_{0}$ is the constant appearing in Theorem 2.1. Then

$$
\left\|\nabla \rho_{\varepsilon}\right\|_{L^{\infty}\left(B_{1 / 2}\right)} \leq C \varepsilon\left(1+E_{\varepsilon}\left(u_{\varepsilon}\right)\right)
$$

where $C$ is a constant depending only on $N$.

Proof. We deduce from Theorem 2.1 and Proposition 2.2 that

$$
\left\|\theta_{\varepsilon}\right\|_{L^{\infty}\left(B_{5 / 6}\right)} \leq C \varepsilon^{2}\left(1+E_{\varepsilon}\left(u_{\varepsilon}\right)\right)
$$

where $\theta_{\varepsilon}=1-\rho_{\varepsilon}$. Hence, going back to (A.5), we have

$$
\left\|a \theta_{\varepsilon}\right\|_{L^{\infty}\left(B_{5 / 6}\right)} \leq C\left(1+E_{\varepsilon}\left(u_{\varepsilon}\right)\right),
$$

so that (A.4), (A.5) and (A.7) yield

$$
\left\|\Delta \theta_{\varepsilon}\right\|_{L^{\infty}\left(B_{5 / 6}\right)} \leq C\left(1+E_{\varepsilon}\left(u_{\varepsilon}\right)\right) .
$$

On the other hand, we have the Gagliardo-Nirenberg inequality

$$
\left\|\nabla \theta_{\varepsilon}\right\|_{L^{\infty}\left(B_{1 / 2}\right)} \leq C\left(\left\|\Delta \theta_{\varepsilon}\right\|_{L^{\infty}\left(B_{5 / 6}\right)}\left\|\theta_{\varepsilon}\right\|_{L^{\infty}\left(B_{5 / 6}\right)}+\left\|\theta_{\varepsilon}\right\|_{L^{\infty}\left(B_{5 / 6}\right)}^{2}\right),
$$

(see e.g. Lemma A.2 in [6]) and the conclusion follows combining (A.34), (A.35) and (A.38).

\section{A.2. Higher integrability}

In the proof of inequality (1.6) and Theorem 1.5 we invoke the following

Theorem A.7. Let $u_{\varepsilon}$ be a solution of $(G L)_{\varepsilon}$ on $B_{1}$. There exists constants $\eta_{1}>0$ and $C>0$ such that if $E_{\varepsilon}\left(u_{\varepsilon}\right) \leq \eta_{1}|\log \varepsilon|$, then

$$
\left(\int_{B_{1 / 2}}|\nabla u|^{2}\right)^{1 / 2} \leq C \int_{B_{1}}|\nabla u| .
$$


Proof. In view of the Clearing-Out property, there exists some constant $\eta_{1}>0$ such that, if

$$
E_{\varepsilon}\left(u_{\varepsilon}\right) \leq \eta_{1}|\log \varepsilon|
$$

then

$$
\left|u_{\varepsilon}\right| \geq 1-\sigma_{0} \quad \text { on } \quad B_{7 / 8} .
$$

For this choice of $\eta_{1}$, we may therefore write on $B_{7 / 8} u_{\varepsilon}=\rho_{\varepsilon} \exp \left(i \varphi_{\varepsilon}\right)$.

Step 1: estimate for the phase. The phase $\varphi_{\varepsilon}$ satisfies the equation

$$
\operatorname{div}\left(\rho_{\varepsilon}^{2} \nabla \varphi_{\varepsilon}\right)=0 \quad \text { on } \quad B_{7 / 8} .
$$

Expanding the differential operator, we are led to

$$
-\Delta \varphi_{\varepsilon}+c \nabla \varphi_{\varepsilon}=0 \quad \text { on } \quad B_{7 / 8}
$$

where we have set $c=-\frac{\nabla \rho_{\varepsilon}}{\rho_{\varepsilon}}$. In view of Theorem A.6, we derive the bound

$$
\|c\|_{L^{\infty}\left(B_{7 / 8}\right)} \leq C \varepsilon^{2}|\log \varepsilon|,
$$

so that, by standard elliptic estimates for (A.42) we have

$$
\left\|\nabla \varphi_{\varepsilon}\right\|_{L^{\infty}\left(B_{6 / 7)}\right)} \leq C \int_{B_{7 / 8}}\left|\nabla u_{\varepsilon}\right| .
$$

Step 2: estimate for the modulus. In this step, we will derive similarly the bound

$$
\left\|\nabla \rho_{\varepsilon}\right\|_{L^{2}\left(B_{1 / 2}\right)} \leq C\|\nabla u\|_{L^{1}\left(B_{1}\right)}
$$

which, combined with (A.43), will yield the conclusion. In order to prove (A.44) we distinguish two cases.

Case A: $\|\nabla u\|_{L^{1}\left(B_{1}\right)} \geq 1$. This is the easiest case. Let $0 \leq \chi \leq 1$ be a smooth cutoff function with compact support in $B_{6 / 7}$ and such that $\chi \equiv 1$ on $B_{5 / 6}$. Multiplying equation (A.4) by $\chi^{2} \theta_{\varepsilon}$ we are led to

$\int_{B_{5 / 6}}\left|\nabla \theta_{\varepsilon}\right|^{2}+\varepsilon^{-2} \theta_{\varepsilon}^{2} \leq C(\chi)\left(\|b\|_{L^{\infty}\left(B_{6 / 7}\right)}\left\|\theta_{\varepsilon}\right\|_{L^{\infty}\left(B_{6 / 7}\right)}+\left\|\nabla \theta_{\varepsilon}\right\|_{L^{\infty}\left(B_{1}\right)}\left\|\theta_{\varepsilon}\right\|_{L^{\infty}\left(B_{6 / 7}\right)}\right)$.

Since $\theta_{\varepsilon}=1-\rho_{\varepsilon}$, we have $\left|\theta_{\varepsilon}\right| \leq C$ by Proposition 2.2, and in view of (A.43) we have $\|b\|_{L^{\infty}\left(B_{6 / 7}\right)} \leq C\left\|\nabla u_{\varepsilon}\right\|_{L^{\infty}\left(B_{1}\right)}^{2}$. Hence, we are led to

$$
\begin{aligned}
\left\|\nabla \theta_{\varepsilon}\right\|_{L^{2}\left(B_{5 / 6}\right)}^{2}+\varepsilon^{-2}\left\|\theta_{\varepsilon}\right\|_{L^{2}\left(B_{5 / 6}\right)}^{2} & \leq C\left(\left\|\nabla u_{\varepsilon}\right\|_{L^{1}\left(B_{1}\right)}\left\|\nabla u_{\varepsilon}\right\|_{L^{1}\left(B_{1}\right)}^{2}\right) \\
& \leq C\left\|\nabla u_{\varepsilon}\right\|_{L^{1}\left(B_{1}\right)}^{2}
\end{aligned}
$$


where we have used the assumption $\|\nabla u\|_{L^{1}\left(B_{1}\right)} \geq 1$; the conclusion (A.44) follows in this case.

Case B : $\|\nabla u\|_{L^{1}\left(B_{1}\right)} \leq 1$. In view of (A.43) and (A.45), we have $\left\|\nabla u_{\varepsilon}\right\|_{L^{2}\left(B_{5 / 6}\right)} \leq$ $C$, and we deduce from Theorem 2.1 and Proposition 2.2 that

$$
\left\|\theta_{\varepsilon}\right\|_{L^{\infty}\left(B_{4 / 5}\right)} \leq C \varepsilon^{2} .
$$

In particular, setting $\alpha(x)=a(x)-2 \varepsilon^{-2}$ we have

$$
\|\alpha\|_{L^{\infty}\left(B_{4 / 5}\right)} \leq C .
$$

Next, let $r \in(3 / 4,4 / 5)$. Multiplying (A.4) by 1 and integrating over $B_{r}$ we obtain

$$
\left|\int_{B_{r}} 2 \varepsilon^{-2} \theta_{\varepsilon}\right| \leq \int_{B_{r}}|b|+\int_{\partial B_{r}}\left|\frac{\partial \theta_{\varepsilon}}{\partial n}\right|+C \int_{B_{r}} \varepsilon^{-2} \theta_{\varepsilon}^{2} .
$$

In particular we may choose some $r$ such that

$$
2 \varepsilon^{-2}\left|\int_{B_{r}} \theta_{\varepsilon}\right| \leq C\left(\left\|\nabla \theta_{\varepsilon}\right\|_{L^{1}\left(B_{1}\right)}+\left\|\nabla u_{\varepsilon}\right\|_{L^{1}\left(B_{1}\right)}\right) \leq C\left\|\nabla u_{\varepsilon}\right\|_{L^{1}\left(B_{1}\right)} .
$$

Therefore, we obtain by Sobolev embedding

$$
\left\|\theta_{\varepsilon}\right\|_{W^{1,1}\left(B_{r}\right)} \leq C\left\|\nabla u_{\varepsilon}\right\|_{L^{1}\left(B_{1}\right)} .
$$

Going back once more to (A.4), we decompose on $B_{3 / 4}$ the function $\theta_{\varepsilon}$ as $\theta_{\varepsilon}=$ $\theta_{\varepsilon}^{0}+\theta_{\varepsilon}^{1}$, where $\theta_{\varepsilon}^{1}$ is the solution of

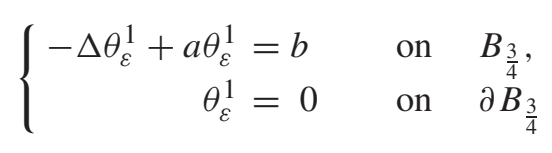

so that $\theta_{\varepsilon}^{0}$ verifies the equation

$$
-\Delta \theta_{\varepsilon}^{0}+a \theta_{\varepsilon}^{0}=0 \quad \text { on } \quad B_{\frac{3}{4}} .
$$

Multiplying (A.48) by $\theta_{\varepsilon}^{1}$, we obtain

$$
\left\|\nabla \theta_{\varepsilon}^{1}\right\|_{L^{2}\left(B_{3 / 4}\right)}^{2}+\varepsilon^{-2}\left\|\theta_{\varepsilon}^{1}\right\|_{L^{2}\left(B_{3 / 4}\right)}^{2} \leq C\left\|\nabla u_{\varepsilon}\right\|_{L^{1}\left(B_{1}\right)}^{2},
$$

so that

$$
\left\|\theta_{\varepsilon}^{1}\right\|_{H^{1}\left(B_{3 / 4}\right)} \leq C|| \nabla u_{\varepsilon} \|_{L^{1}\left(B_{1}\right)} .
$$

We claim that similarly, we have the estimate for $\theta_{\varepsilon}^{0}$

$$
\left\|\nabla \theta_{\varepsilon}^{0}\right\|_{L^{2}\left(B_{1 / 2}\right)} \leq C\left\|\theta_{\varepsilon}^{0}\right\|_{W^{1,1}\left(B_{3 / 4}\right)},
$$


where the constant $C$ is independent of $\varepsilon$. We postpone the proof of (A.51) and complete the proof of (A.43) in Case B. Combining (A.50) and (A.51), we have

$$
\begin{aligned}
\left\|\nabla \rho_{\varepsilon}\right\|_{L^{2}\left(B_{1 / 2}\right)} & \leq\left\|\nabla \theta_{\varepsilon}^{1}\right\|_{L^{2}\left(B_{1 / 2}\right)}+\left\|\nabla \theta_{\varepsilon}^{0}\right\|_{L^{2}\left(B_{1 / 2}\right)} \\
& \leq C\left(\left\|\nabla u_{\varepsilon}\right\|_{L^{1}\left(B_{1}\right)}+\left\|\theta_{\varepsilon}-\theta_{\varepsilon}^{1}\right\|_{W^{1,1}\left(B_{3 / 4}\right)}\right) \\
& \leq C\left(\left\|\nabla u_{\varepsilon}\right\|_{L^{1}\left(B_{1}\right)}+\left\|\theta_{\varepsilon}\right\|_{W^{1,1}\left(B_{3 / 4}\right)}+\left\|\theta_{\varepsilon}^{1}\right\|_{H^{1}\left(B_{3 / 4}\right)}\right) \\
& \leq C\left\|\nabla u_{\varepsilon}\right\|_{L^{1}\left(B_{1}\right)}
\end{aligned}
$$

where we have used (A.50) for the third inequality. Inequality (A.43) is therefore established.

Proof of the claim (A.51). Let $0 \leq \chi \leq 1$ be a smooth cut-off function with compact support in $B_{3 / 4}$, and consider the operator $L_{\varepsilon}=-\Delta+2 \varepsilon^{-2}$ on $\mathbb{R}^{N}$. We have, for $\theta_{\varepsilon}^{0, \chi}=\chi \theta_{\varepsilon}^{0}$,

$$
L_{\varepsilon}\left(\theta_{\varepsilon}^{0, \chi}\right)=f_{\varepsilon}^{\chi} \equiv 2 \nabla \chi \nabla \theta_{\varepsilon}^{0}+(\Delta \chi-\alpha \chi) \theta_{\varepsilon}^{0}
$$

Notice that for any $1 \leq p \leq+\infty$, we have

$$
\left\|f_{\varepsilon}\right\|_{L^{p}\left(\mathbb{R}^{N}\right)} \leq C(\chi)\left\|\theta_{\varepsilon}^{0}\right\|_{W^{1, p}(\operatorname{supp} \chi)} .
$$

The kernel $K_{\varepsilon}$ associated to the operator $L_{\varepsilon}$ is given by

$$
K_{\varepsilon}(x)=c_{N}|x|^{2-N} \exp \left(-\frac{|x|}{\varepsilon}\right) \quad \text { if } N \geq 3
$$

and

$$
K_{\varepsilon}(x)=c_{2} \log (|x|) \exp \left(-\frac{|x|}{\varepsilon}\right) \quad \text { if } N=2 .
$$

In particular, we have for any $1 \leq r<\frac{N}{N-1}$

$$
\left\|K_{\varepsilon}\right\|_{W^{1, r}\left(\mathbb{R}^{N}\right)} \leq C(r),
$$

where the constant $C(r)$ is independent of $\varepsilon$. It follows that for every $1 \leq q<p^{*} \equiv$ $\frac{N p}{N-p}$

$$
\left\|\theta_{\varepsilon}^{0, \chi}\right\|_{W^{1, q}\left(\mathbb{R}^{N}\right)} \leq C(\chi, q)\left\|\theta_{\varepsilon}^{0}\right\|_{W^{1, p}(\operatorname{supp} \chi)} .
$$

We iterate the previous inequality for suitable choices of cut-off functions $\chi$ and $q=1^{*}, 1^{* *}, \cdots$ up to $q=2$. This yields

$$
\mid \theta_{\varepsilon}^{0}\left\|_{H^{1}\left(B_{1 / 2}\right)} \leq C\right\| \theta_{\varepsilon}^{0} \|_{W^{1,1}\left(B_{1}\right)} .
$$




\section{References}

[1] D. Adams, A Trace inequality for generalized potentials, Studia Math. 48 (1973), 99-105.

[2] D. AdAMS, Weighted nonlinear potential theory, Trans. Amer. Math. Soc. 297 (1986), $73-$ 94.

[3] G. Alberti, S. BAldo and G. ORLAndi, Variational convergence for functionals of Ginzburg-Landau type, Indiana Univ. Math. J., to appear

[4] L. Ambrosio and M. Soner, A measure theoretic approach to higher codimension mean curvature flow, Ann. Scuola Norm. Sup. Pisa Cl. Sci. 25 (1997), 27-49.

[5] F. Bethuel, J. Bourgain, H. Brezis and G. Orlandi, $W^{1, p}$ estimates for solutions to the Ginzburg-Landau equations with boundary data in $H^{1 / 2}$, C.R. Acad. Sci. Paris Sér. I Math. 333 (2001), 1-8.

[6] F. Bethuel, H. Brezis and F. HéLein, Asymptotics for the minimization of a GinzburgLandau functional, Calc. Var. Partial Differential Equations 1 (1993), 123-148.

[7] F. Bethuel, H. Brezis and F. HÉLein, "Ginzburg-Landau vortices", Birkhäuser, Boston, 1994.

[8] F. Bethuel, H. Brezis and G. ORLAndi, Asymptotics for the Ginzburg-Landau equation in arbitrary dimensions, J. Funct. Anal. 186 (2001), 432-520. Erratum 188 (2002), 548549.

[9] F. Bethuel and G. ORLAndi, Uniform estimates for the parabolic Ginzburg-Landau equation, ESAIM Control Optim. Calc. Var. 9 (2002), 219-238.

[10] F. Bethuel, G. ORLANDi and D. Smets, Vortex rings for the Gross-Pitaevskii equation, J. Eur. Math. Soc. 6 (2004), 17-94.

[11] F. Bethuel, G. ORLAndi and D. Smets, Convergence of the parabolic Ginzburg-Landau equation to motion by mean curvature, Ann. of Math., to appear

[12] F. Bethuel, G. ORLAndi and D. SMets, Motion of concentration sets in GinzburgLandau equations, Ann. Sci. Fac. Toulouse 13 (2004), 3-43.

[13] F. Bethuel, G. ORLANDI and D. SMets, Approximations with vorticity bounds for the Ginzburg-Landau functional, Commun. Contemp. Math. 6 (2004), 803-832.

[14] F. Bethuel, G. ORlandi and D. Smets, Collisions and phase-vortex interactions in dissipative Ginzburg-Landau dynamics, Duke Math. J., to appear.

[15] F. BETHUEL and T. Rivière, A minimization problem related to superconductivity, Ann. Inst. H. Poincaré Anal. Non Linéaire 12 (1995), 243-303.

[16] J. BouRGAIN and H. BREZIS, New estimates for the Laplacian, the div-curl and related Hodge systems, C.R. Acad. Sci. Paris Sér. I Math. 338 (2004), 539-543.

[17] J. Bourgain, H. Brezis and P. Mironescu, On the structure of the Sobolev space $H^{1}$ with values in the circle, C.R. Acad. Sci. Paris Série I 331 (2000), 119-124.

[18] J. Bourgain, H. Brezis and P. Mironescu, $H^{1 / 2}$ maps with values into the circle: minimal connections, lifting and the Ginzburg-Landau equation, Inst. Hantes Études Sci. Publ. Math. 99 (2004), 1-115.

[19] K. BRAKKE, "The motion of a surface by its mean curvature", Princeton University Press, Princeton, 1978.

[20] H. Brezis and P. MironesCu, Sur une conjecture de E. De Giorgi relative à l'énergie de Ginzburg-Landau, C. R. Acad. Sci. Paris Série I Math. 319 (1994), 167-170.

[21] Y. Chen and M. STRUWE, Existence and partial regularity results for the heat flow for harmonic maps, Math. Z. 201 (1989), 83-103.

[22] D. Chiron, Boundary problems for the Ginzburg-Landau equation, Comm. Contemp. Math., to appear.

[23] R. Coifman, P. L. Lions, Y. Meyer and S. Semmes, Compensated compactness and Hardy spaces J. Math. Pures Appl. 72 (1993), 247-286.

[24] M. COMTE and P. Mironescu, The behavior of a Ginzburg-Landau minimizer near its zeroes, Calc. Var. Partial Differential Equations 4 (1996), 323-340. 
[25] R. HERVÉ, and M. HERVÉ, Etude qualitative des solutions réelles d'une équation différentielle liée à l'équation de Ginzburg-Landau, Ann. Inst. H. Poincaré Anal. Non Linéaire 11 (1994), 427-440.

[26] L. HedBerg and T. WolfF, Thin sets in nonlinear potential theory, Ann. Inst. Fourier (Grenoble) 23 (1983), 161-187.

[27] T. ILMANEN, Convergence of the Allen-Cahn equation to Brakke's motion by mean curvature, J. Differential Geom. 38 (1993), 417-461.

[28] R. L. JERRARD and H. M. SONER, The Jacobian and the Ginzburg-Landau energy, Calc. Var. Partial Differential Equations 14 (2002), 151-191.

[29] F. H. LIN and T. Rivière, Complex Ginzburg-Landau equations in high dimensions and codimension two area minimizing currents, J. Eur. Math. Soc. 1 (1999), 237-311. Erratum, J. Eur. Math. Soc. 2 (2000), 87-91.

[30] F. H. LIN and T. Rivière, A quantization property for static Ginzburg-Landau vortices, Comm. Pure Appl. Math. 54 (2001), 206-228.

[31] B. MUCKENHOUPT and R. WHEEDEN, Weighted norm inequalities for fractional integrals, Trans. Amer. Math. Soc. 192 (1974), 261-274.

[32] D. PREISs, Geometry of measures in $\mathbb{R}^{n}$ : distribution, rectifiability, and densities, Ann. of Math. 125 (1987), 537-643.

[33] T. RIVIÈRE, Line vortices in the U(1) Higgs model, ESAIM Control Optim. Calc. Var. 1 (1996), 77-167.

[34] R. SCHOEN and S. T. YAU, "Lectures on harmonic maps", International Press, Cambridge, MA, 1997.

[35] L. Simon, Lectures on Geometric Measure Theory, Proc. of the centre for Math. Anal., Austr. Nat. Univ., 1983.

[36] M. STRUwe, On the asymptotic behavior of minimizers of the Ginzburg-Landau model in 2 dimensions, Differential Integral Equations 7 (1994), 1613-1624.

[37] J. VAN SCHAFTINGEN, A simple proof of an inequality of Bourgain, Brezis and Mironescu, C.R. Acad. Sci. Paris, Sér. 1 Math. 338 (2004), 23-26.

[38] W. P. ZIEMER, "Weakly differentiable functions. Sobolev spaces and functions of bounded variation", Graduate Texts in Math. 120, Springer Verlag, New York, 1989.

Laboratoire Jacques-Louis Lions

Université de Paris 6

4 place Jussieu BC 187

75252 Paris, France

bethuel@ann.jussieu.fr

Dipartimento di Informatica

Università di Verona

strada le Grazie

37134 Verona, Italy

orlandi@sci.univr.it

Centro di Ricerca Matematica Ennio De Giorgi

Scuola Normale Superiore di Pisa

Piazza dei Cavalieri 3

56100 Pisa, Italy

smets@ann.jussieu.fr 